\title{
Modeling and analysis of magnetic hybrid
}

\section{nanoparticle $\left(\mathrm{Au}-\mathrm{Al}_{2} \mathrm{O}_{3} /\right.$ blood $)$ based drug delivery through a}

\section{bell-shaped occluded artery with Joule heating, viscous dissipation and variable viscosity effects}

\author{
Rishu Gandhi*1 ${ }^{*}$ B. K. Sharma ${ }^{\dagger 1}$, Chandan Kumawat ${ }^{\ddagger 1}$, and O. Anwar Beg ${ }^{\S 2}$ \\ ${ }^{1}$ Department of Mathematics, Birla Institute of Technology and Science, Pilani, Rajasthan, India \\ ${ }^{2}$ Multi-Physical Engineering Sciences Group (MPESG), Department of Mechanical and Aeronautical \\ Engineering, School of Science, Engineering and Environment (SEE), Newton Building-G77/G84, Salford \\ University, The Crescent, Manchester, M54WT, UK
}

\begin{abstract}
The present work deals with the impact of hybrid nanoparticles $\left(\mathrm{Au}-\mathrm{Al}_{2} \mathrm{O}_{3} / \mathrm{Blood}\right)$ on the blood flow pattern through a porous cylindrical artery with bell-shaped stenosis in the presence of an external magnetic field, Joule heating, and viscous dissipation by considering twodimensional pulsatile blood flow. The temperature-dependent viscosity model is utilized in this model. The blood flow is assumed to be unsteady, laminar, viscous, and incompressible. The mild stenotic presumption normalizes and reduces the bi-directional flow to uni-directional. The Crank-Nicolson scheme is applied to solve the continuity, momentum, and energy equations with appropriate initial and boundary conditions. The acquired results of the work are
\end{abstract}

\footnotetext{
*rishugandhi155@gmail.com

†Corresponding Author: bhupen_1402@yahoo.co.in

†chandankumawat000@gmail.com

§o.a.beg@salford.ac.uk
} 
presented graphically. They have been examined for several values of the dimensionless parameters such as Magnetic number $\left(M^{2}\right)$, Darcy number $(D a)$, Grashof number $(G r)$, viscosity parameter $\left(\beta_{0}\right)$, Reynolds number $(R e)$, Eckert Number $(E c)$, Prandtl number $(P r)$, different concentration of both the nanoparticles $\left(\phi_{1}, \phi_{2}\right)$, and pressure gradient parameter $\left(B_{1}\right)$. The velocity contours for different emerging parameters have been drawn to analyze the overall behavior of blood flow patterns. The non-dimensional velocity profile enhances with increment in values of $D a$, implying that the medium's permeability provides less barrier to flow. The cumulative impact of Joule Heating and viscous dissipation are discussed. It demonstrates that increasing viscous dissipation $(E c)$ and Joule heating $\left(M^{2}\right)$ parameter simultaneously raise the nanofluid temperature since the mechanical energy is transformed to thermal energy within molecules, which causes a hike in temperature. The findings reveal that hybrid nanoparticles $\left(\mathrm{Au}-\mathrm{Al}_{2} \mathrm{O}_{3}\right.$ /blood) effectively reduce hemodynamic variables such as wall shear stress and resistance impedance. Results indicate that nanoparticles may be helpful to keep the blood velocity under control and allow the surgeons to adjust it as and when required. The present work aims to get insight into the treatment of atherosclerosis without surgery, lower medical costs, and reduce post-surgical complications. Also, it has broad implications in treating various conditions, including cancers, infections, and the removal of blood clots. The current findings are consistent with recent findings in earlier blood flow research studies.

Keywords: MHD, heat transfer, bell-shaped stenosis, temperature-dependent viscosity, drug delivery.

\section{Introduction}

A nanoparticle is a tiny particle undetectable by the human eye, and its size ranges between 1 to 100 nanometers. Nanoparticles can occur naturally and be created in laboratories with the desired properties. They can exhibit different physical and chemical properties. In contrast to bulk mate- 


\begin{tabular}{|c|c|}
\hline Nomenclature & $\begin{array}{ll}t_{1}^{*} & \text { Time }(s) \\
\tilde{T}^{*} & \text { Temperature of the base fluid }(K)\end{array}$ \\
\hline$B_{0}$ Uniform Magnetic Field $(T)$ & $\tilde{T}_{1}^{*}$ Reference temperature $(K)$ \\
\hline$B_{1} \quad$ Pressure gradient parameter $(\mathrm{Pa} / \mathrm{m})$ & $\tilde{T}_{w}^{*}$ Temperature at the wall $(K)$ \\
\hline$\tilde{C}_{p}^{*} \quad$ Specific heat at constant pressure $\left(J k g^{-1} K^{-1}\right)$ & $u_{1}^{*}$ Velocity component in radial direction $(\mathrm{m} / \mathrm{s})$ \\
\hline$D a^{-1}$ Inverse Darcy Number (-) & $U_{0}$ Reference velocity $(\mathrm{m} / \mathrm{s})$ \\
\hline Ec Eckert Number (-) & $w_{1}^{*}$ Velocity component in axial direction $(\mathrm{m} / \mathrm{s})$ \\
\hline g Acceleration by virtue of gravity $\left(\mathrm{m} / \mathrm{s}^{2}\right)$ & $z_{1}^{*} \quad$ Axial direction $(m)$ \\
\hline Gr Grashof Number (-) & Greek Letters \\
\hline$k_{1}^{*} \quad$ Permeability of the porous medium $\left(\mathrm{m}^{2}\right)$ & $\beta_{0}$ Viscosity parameter (-) \\
\hline$k_{f} \quad$ Thermal conductivity $(W /(m K))$ & $\gamma \quad$ Thermal expansion coefficient $\left(K^{-1}\right)$ \\
\hline$M^{2} \quad$ Hartmann Number (-) & $\delta \quad$ Stenosis depth $(m)$ \\
\hline$p_{1}^{*} \quad$ Pressure $(P a)$ & $\tilde{\theta} \quad$ Non-dimensional temperature (-) \\
\hline $\operatorname{Pr} \quad$ Prandtl Number (-) & $\lambda$ Resistance Impedance $\left(\right.$ Pa.s $\left./ \mathrm{m}^{3}\right)$ \\
\hline$q_{w} \quad$ Heat transfer Coefficient $\left(W /\left(m^{2} K\right)\right)$ & $\mu_{0} \quad$ Reference viscosity (Pa.s) \\
\hline$Q_{1} \quad$ Flow Rate $\left(\mathrm{m}^{3} / \mathrm{s}\right)$ & $\mu_{f}$ Blood's viscosity $($ Pa.s) \\
\hline$r_{1}^{*} \quad$ Radial direction $(m)$ & $\rho$ Density $\left(\mathrm{kg} / \mathrm{m}^{3}\right)$ \\
\hline $\mathrm{R} \quad$ Radius of artery in stenotic region $(m)$ & $\sigma$ Electrical conductivity $(S / m)$ \\
\hline$R_{0} \quad$ Radius of artery in non-stenotic region $(\mathrm{m})$ & $\tau_{w}$ Shear stress at the wall $(\mathrm{Pa})$ \\
\hline Re Reynolds Number (-) & $\phi \quad$ Nanoparticle Concentration $\left(\mathrm{mol} / \mathrm{m}^{3}\right)$ \\
\hline
\end{tabular}

rials, they have a high surface-to-volume ratio. Nanomaterials are used in various fields, including healthcare, the environment, and cosmetics. Drug delivery is one of the many applications of nanomaterials in healthcare. One example of this technology is developing nanoparticles to help carry chemotherapy drugs directly to malignant growths and provide pharmaceuticals to damaged regions of arteries to address cardiovascular disease. Ijaz et al. [1] addressed how $\mathrm{Cu}$-nanoparticles affect the blood flow through a porous artery with composite stenosis. Using an appropriate mathematical model, Nadeem et al. [2] examined the impact of nanoparticles on blood flow properties through an artery with bell-shaped stenosis. Eid et al. [3] discusses the heat transfer characteristics of gold nanoparticles with Sisko bio-nanofluid flow subject to non-linear thermal radiation. To characterize the dynamical behavior of the blood, Zaman et al. [4] considered Carreau fluid model and incorporated the effects of nanoparticles mathematically employing Buonjiornio's formulation. Ayub et al. [5] studied the flow of blood mathematically in a curved stenotic artery along with a balloon regarding blood as a viscous fluid. This exploratory research aimed to look at 
the applications of $\mathrm{Cu}$-blood nanofluid in overlapping stenosis. Chatterjee et al. [6] contemplated Bernstein polynomial approximation to depict Au-blood nanofluid transport characteristics via a stenosed artery subject to the magnetic field. Tripathi et al. [7] presented a thermal examination of $\mathrm{Cu}-\mathrm{CuO} / \mathrm{blood}$ nanofluids flow in a microvessel considering nanoparticle shape effects. Elkhair et al. [8] investigated the effect of magnetic force and non-linear thermal radiation considering gold and copper nanoparticles on hybrid bio-nanofluid flow subject to an applied magnetic field. Khan et al. [9] investigated bioconvection in Oldroyd-B nanofluid using a convectively heated surface considering partial slip effects. Jamshed et al. [10] explored thermal transfer to enrich the research of the solar aircraft wings by using hybrid nanofluid via parabolic trough solar collector (PTSC). Hybrid nanofluids have been a prominent topic of study for several researchers ([11, 12, 13, 14]).

Blood is a convoluted fluid. Blood flow into stenotic arteries has attracted much interest from researchers over the last few decades and has developed into an appealing research subject. The intermittent pumping action of the heart causes the pulsatile character of blood flow in arteries. With the aid of numerical simulation, Alimohamadi et al. [15] investigated pulsatile blood flow subject to a transient magnetic field through a coupled free and porous media, which was overlooked in previous studies. The impact of nanoparticles $\left(\mathrm{Cu}, \mathrm{TiO}_{2}, \mathrm{Al}_{2} \mathrm{O}_{3}\right)$ through a curved stenosed channel on unsteady pulsatile blood flow was studied by Zaman et al. [16]. Varmazyar et al. [17] performed numerical simulation and investigated the impact of magneto-nanoparticles distribution on atherosclerotic plaques. Somasundaram and Reddy [18] used the Maxwell Garnett model for nanofluid thermal conductivity to investigate the MHD pulsing flow of blood-carrying $\mathrm{Al}_{2} \mathrm{O}_{3}$ nanoparticles in a channel with thermal radiation, viscous dissipation, and Joule heating effects.

The viscosity of blood in a biological system is not constant and is affected by various factors, including vessel width, axial or radial coordinates, temperature, and hematocrit ratio. In reality, as blood circulation increases, blood thickness decreases, maximizing blood flow and lowering coagulation factors. Synder et al. [19] analyzed the temperature effects on viscosity of blood us- 
ing glass tube viscometers. Since lizards are naturally exposed to temperature changes, they were chosen for this research. Eckmann [20] tried to figure out the effects of temperature, hematocrit, and shear rate on viscosity of blood in conditions that resembled profound hypothermia for cardiac surgery. The transport of nanoparticles via a stenosed artery with porous walls and variable viscosity was examined by Akbar et al. [21]. Tiwari et al. [22] examined how dependency of viscosity on hematocrit affects blood flow via a narrow tube.

MHD is a physical-mathematical system that studies the dynamics of magnetic fields in electrically conducting fluids, such as plasmas and liquid metals. The basic idea of MHD theory is that conductive fluids can support magnetic fields. Magnetic fields produce forces that act on the fluid (usually plasma), potentially changing the geometry and strength of the magnetic fields. Bhatti et al. [23] explored the MHD effects on peristaltic blood flow of the Jeffrey fluid model in a porous channel. Mirza et al. [24] used a magneto-hydrodynamic approach to investigate non-Newtonian blood flow in a stenosed artery in conjunction with magnetic particles. Abdollahzadeh et al. [25] employed the Carreau formulation to depict the non-Newtonian behavior in a stenosed artery. The developed model incorporates both ferro-hydrodynamic (FHD) and magnetohydrodynamic (MHD) concepts, taking blood conductivity and magnetization into account. The MHD and shape factor effects on a fluid containing hybrid nanoparticles were explored analytically and numerically by Khentout et al. [26]. Jha and Gwandu [27] studied MHD free convective flow down a vertical micro-channel with one channel wall surface having no slip and the other having super-hydrophobic properties. The impact of MHD with various other influential factors such as variable viscosity, Joule heating, radiation, and chemical reaction was studied by Tripathi et al. ([28],[29], [30]).

Porous media has a wide range of applications in a variety of disciplines. Nanofluid is a modern form of working fluid that can be used to improve thermal properties. There are many approaches for modeling nanofluids in a porous medium. One of the more efficient ways is the 
Darcy model used in this article. Beg et al. [31] investigated the micropolar blood flow through a two-dimensional square bound by impermeable walls. Sharma et al. [32] studied non-Newtonian hydromagnetic biofluid flow with the Joule effect in a non-Darcian porous medium. El-Dabe et al. [33] examined the effect of slip on the motion of Sisko fluid by assuming moderate stenosis via a porous material. To show nanofluid MHD flow through a porous enclosure, Sheikholeslami [34] used an innovative numerical approach CVFEM. In the presence of non-linear thermal radiation and heat source/sink, Eid et al. [35] investigated MHD flow through a circular cylinder using three different nanoparticles forms. Bhatti et al. [36] employed the Differential Transform Method (DTM) to analyze the nanofluid flow and microbe swimming via rotating circular plates placed parallelly in porous media. Zhang et al. [37] investigated magnetic nanofluid dynamics with Arrhenius kinetics and Lorentz forces using a non-linear porous stretching sheet. Bilal et al. [38] optimized the entropy of a non-Darcian Williamson nanofluid with non-linear thermal radiation, considering the effects of viscous dissipation, chemical reaction, and Joule heating.

Cardiovascular diseases come in a category of hazardous illnesses for humans. It is mandatory first to understand them properly and then find remedies to cure them. There is a traditional drug delivery system to cure such illnesses, but many side effects come. So, in the modern era, the nanoparticle drug delivery system is more accurate than the traditional drug delivery system. It is accurate in the sense that we can deliver the drug and target the drug with the help of nanoparticles to a precise location. In this way, the side effects are reduced to the extent that the other healthy organs are not affected by medication delivered to the damaged part. In a computational study of unsteady magnetic drug targeting in partially occluded blood arteries, Bose et al. [39] examined the impact of particle-fluid coupling on magnetic particle transport using an Eulerian-Lagrangian approach. ANSYS FLUENT is used to determine the hemodynamic flow and the mobility of the magnetic particles in the flow in this study. Chakravarty [40] depicted the role of mathematical modeling in the elucidation of drug release mechanisms and promoting the creation of new therapeutic drugs through a systematic approach. Ponalagusamy [41] investigated a theoretical model 
of the stenosed artery assuming blood as Herschel-Bulkley fluid-carrying iron oxide nanoparticles under periodic body acceleration. Dinarvand et al. [42] investigated the mixed convection flow of an electrically conducting hybrid nanofluid $(\mathrm{CuO}-\mathrm{Cu} / \mathrm{blood})$ subjected to an external magnetic field and induced magnetic field effects in biomedical applications, especially drug delivery.

Drug delivery with the help of nanoparticles has gained a lot of attention in the past few decades. The main reason behind this is that there are many side effects in the traditional drug delivery system. The amount of the drug received by the diseased organ is less as the drug is distributed uniformly throughout the body. Also, this affects other healthy organs of the human body. This research work is also motivated by the benefits of the nanoparticle drug delivery system. In this work, we have considered the gold $(\mathrm{Au})$ and alumina $\left(\mathrm{Al}_{2} \mathrm{O}_{3}\right)$ nanoparticles and have taken their combination with blood to form $\mathrm{Au}-\mathrm{Al}_{2} \mathrm{O}_{3} / \mathrm{Blood}$ hybrid nanofluid. Present research work is divided into four parts, which are as follows: The first part is the introduction, which includes various physical parameters considered in this work, and the other related work done by researchers. The second part represents mathematical modeling which includes geometrical representation of the model and governing equations. It also consists of the non- dimensionalization part and radial coordinate transformation. Next is the numerical solution part, which includes the numerical scheme and discretization of modified equations. Lastly, there is the results and graphical analysis part. The validation of the work done is necessary, represented in this part. Also, the results are plotted graphically with the help of MATLAB and then elaborated these graphical results.

To the best of our knowledge, no work has yet been done in a bell-shaped stenosed artery using $\mathrm{Au}-\mathrm{Al}_{2} \mathrm{O}_{3}$ hybrid nanoparticles suspended in the blood flow as a porous media considering temperature-dependent viscosity model. Most studies have also disregarded the effects of Joule heating and viscous dissipation. As a result, the work's novelty is analyzing the cumulative impact of Joule heating and viscous dissipation by incorporating $\mathrm{Au}-\mathrm{Al}_{2} \mathrm{O}_{3}$ nanoparticles in the blood flow as a porous media on hemodynamical quantities such as wall shear stress, flow rate, resis- 
tance impedance, heat transfer coefficient for an occluded bell-shaped artery. The present work aims to get insight into the treatment of atherosclerosis without surgery, lower medical costs, and reduce post-surgical complications. Also, it has broad implications in treating various conditions, including cancers, infections, and the removal of blood clots.

\section{Mathematical Modeling}

In this article, an axisymmetric artery with bell-shaped stenosis is under consideration, and the blood flow through the artery is assumed to be unsteady, laminar, viscous, and incompressible. The mathematical equations are expressed in a cylindrical coordinate system $\left(r_{1}^{*}, \tilde{\theta}, z_{1}^{*}\right)$, in which the $r_{1}^{*}$ signifies the radial coordinate, $\tilde{\theta}$ is a circumferential (azimuthal) coordinate, and $z_{1}^{*}$ is an axial (longitudinal) coordinate, respectively. The radial and axial components of velocity are $u_{1}^{*}$ and $w_{1}^{*}$, respectively. The flow is assumed to be independent of $\tilde{\theta}$ due to the axisymmetry of the artery. A uniform magnetic field $B_{0}$ is applied perpendicular to the axial direction of the blood flow. The strength of the induced magnetic field is very small in contrast to the applied magnetic field strength due to the low electrical conductivity of blood. Therefore, the induced magnetic field has been neglected in the current analysis. The impact of heat transfer phenomena in blood flow has also been considered. The thermal energy equation includes viscous dissipation due to the incorporation of the temperature-dependent variable viscosity. The blood flowing through an occluded bell-shaped stenosed artery is injected with gold $(\mathrm{Au})$ and alumina $\left(\mathrm{Al}_{2} \mathrm{O}_{3}\right)$ nanoparticles.

\subsection{Geometrical Representation of the model}

The mathematical equation that represents the geometry of the artery [43, 44] is:

$$
R\left(z_{1}^{*}\right)= \begin{cases}{\left[R_{0}-\delta \operatorname{Exp}\left(\frac{-m^{2} \epsilon^{2}}{R_{0}^{2}}\left(z_{1}^{*}-\frac{3 l_{0}}{2}\right)^{2}\right)\right]} & d \leq z_{1}^{*} \leq d+l_{0} \\ R_{0} & \text { otherwise }\end{cases}
$$


where $\mathrm{R}\left(z_{1}^{*}\right)$ represents the radius of the stenosed portion of the arterial segment, $R_{0}$ is the radius of the normal artery in the non-stenotic region, $l_{0}$ is the length of the stenosis, $\mathrm{d}$ is the location of the stenosis, $\mathrm{m}$ is a parametric constant, $\epsilon$ characterizes the relative length of the constriction, defined as the ratio of the radius to the length of the stenosis, $\delta$ is the critical height of the stenosis. Figure (1a) highlights the geometry of the artery with suspended nanoparticles and blood cells, whereas the combination of nanoparticles used in this study is depicted in pictorial form as given in Figure (1b).
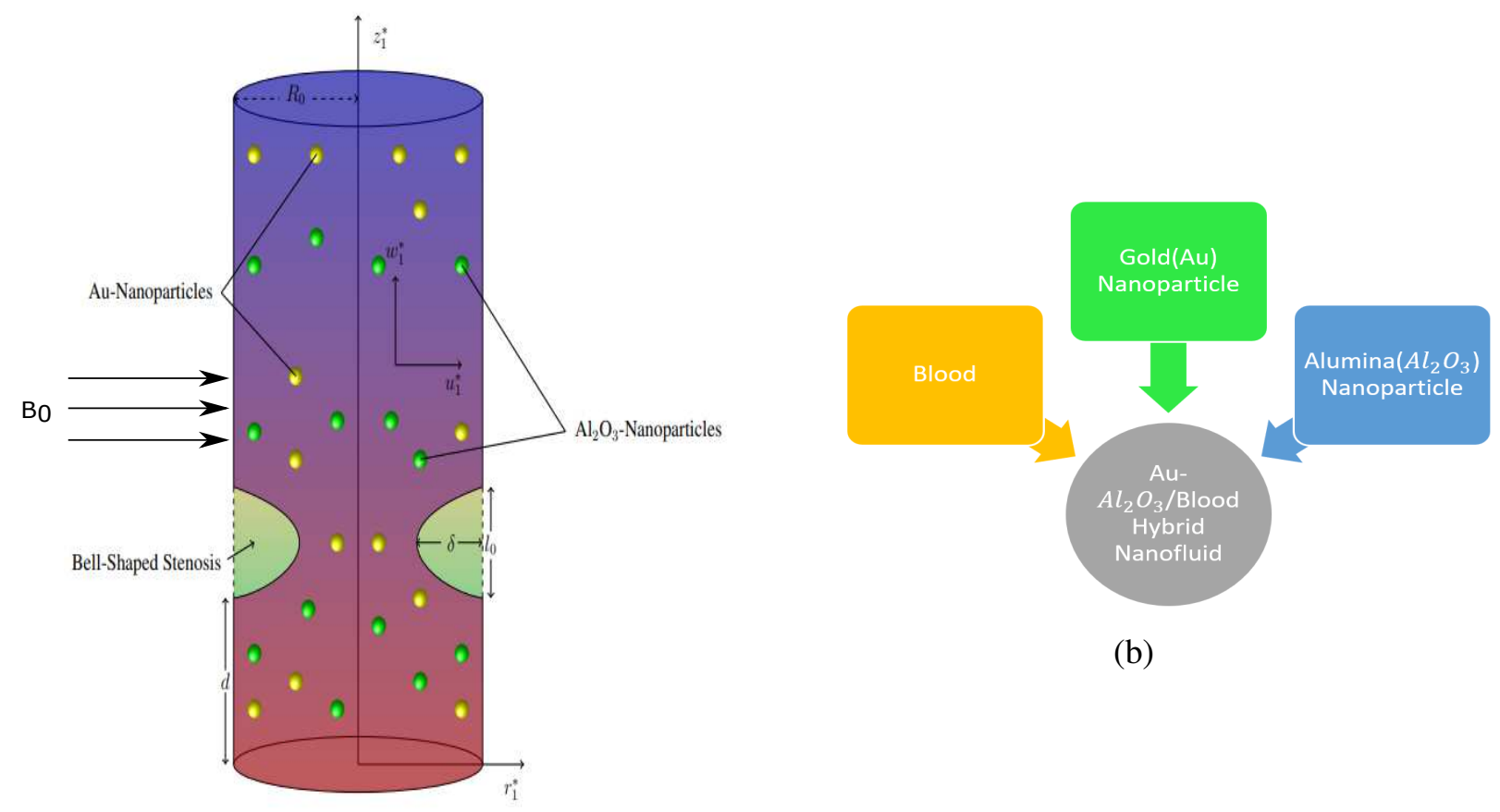

(b)

(a)

Figure 1: (a) The physical sketch of the bell-shaped constricted artery with nanoparticles, (b) Combination of nanoparticles with blood as base fluid to form hybrid nanofluid

\subsection{Governing Equations}

The velocity and temperature fields for the present study are defined as follows in the case of unsteady and axisymmetric hybrid nanoparticle-doped blood flow:

$$
\tilde{V}^{*}=\tilde{v}^{*}\left[u_{1}^{*}\left(r_{1}^{*}, z_{1}^{*}, t_{1}^{*}\right), 0, w_{1}^{*}\left(r_{1}^{*}, z_{1}^{*}, t_{1}^{*}\right)\right], \quad \tilde{T}^{*}=\tilde{T}^{*}\left(r_{1}^{*}, z_{1}^{*}, t_{1}^{*}\right)
$$


where $u_{1}^{*}$ and $w_{1}^{*}$ are the radial and axial components respectively.

The flow is assumed to be governed by Darcy's law, modified by buoyancy, and subject to the Boussinesq approximation. Thus, for buoyancy-driven convection, the governing equations for fluid motion and temperature field are given as ([45],[46],[47]):

\section{Continuity Equation:}

$$
\frac{\partial u_{1}^{*}}{\partial r_{1}^{*}}+\frac{u_{1}^{*}}{r_{1}^{*}}+\frac{\partial w_{1}^{*}}{\partial z_{1}^{*}}=0
$$

\section{Momentum Equation:}

\section{r-direction:}

$$
\begin{array}{r}
\rho_{h n f}\left[\frac{\partial u_{1}^{*}}{\partial t_{1}^{*}}+u_{1}^{*} \frac{\partial u_{1}^{*}}{\partial r_{1}^{*}}+w_{1}^{*} \frac{\partial u_{1}^{*}}{\partial z_{1}^{*}}\right]=-\frac{\partial p_{1}^{*}}{\partial r_{1}^{*}}+\frac{1}{r_{1}^{*}} \frac{\partial}{\partial r_{1}^{*}}\left[\mu_{h n f}\left(\tilde{T}^{*}\right) \frac{\partial u_{1}^{*}}{\partial r_{1}^{*}}\right]+\frac{\partial}{\partial z_{1}^{*}}\left[\mu_{h n f}\left(\tilde{T}^{*}\right)\left(\frac{\partial u_{1}^{*}}{\partial z_{1}^{*}}+\frac{\partial w_{1}^{*}}{\partial r_{1}^{*}}\right)\right] \\
-2 \mu_{h n f}\left(\tilde{T}^{*}\right) \frac{u_{1}^{*}}{r_{1}^{* 2}}, \quad(3)
\end{array}
$$

\section{z-direction:}

$$
\begin{aligned}
\rho_{h n f}\left[\frac{\partial w_{1}^{*}}{\partial t_{1}^{*}}+u_{1}^{*} \frac{\partial w_{1}^{*}}{\partial r_{1}^{*}}+w_{1}^{*} \frac{\partial w_{1}^{*}}{\partial z_{1}^{*}}\right]=-\frac{\partial p_{1}^{*}}{\partial z_{1}^{*}}+\frac{1}{r_{1}^{*}} \frac{\partial}{\partial r_{1}^{*}}\left[r_{1}^{*} \mu_{h n f}\left(\tilde{T}^{*}\right)\left(\frac{\partial u_{1}^{*}}{\partial z_{1}^{*}}+\frac{\partial w_{1}^{*}}{\partial r_{1}^{*}}\right)\right]+ \\
\frac{\partial}{\partial z_{1}^{*}}\left[2 \mu_{h n f}\left(\tilde{T}^{*}\right) \frac{\partial w_{1}^{*}}{\partial z_{1}^{*}}\right]+(\rho \gamma)_{h n f} g\left(\tilde{T}^{*}-\tilde{T}_{1}^{*}\right)-\sigma_{h n f} B_{0}^{2} w_{1}^{*}-\frac{\mu_{h n f}}{k_{1}^{*}} w_{1}^{*},
\end{aligned}
$$

\section{Energy Equation:}

$$
\left(\rho C_{p}\right)_{h n f}\left[\frac{\partial \tilde{T}^{*}}{\partial t_{1}^{*}}+u_{1}^{*} \frac{\partial \tilde{T}^{*}}{\partial r_{1}^{*}}+w_{1}^{*} \frac{\partial \tilde{T}^{*}}{\partial z_{1}^{*}}\right]=k_{h n f}\left[\frac{\partial^{2} \tilde{T}^{*}}{\partial r_{1}^{* 2}}+\frac{1}{r_{1}^{*}} \frac{\partial \tilde{T}^{*}}{\partial r_{1}^{*}}+\frac{\partial^{2} \tilde{T}^{*}}{\partial z_{1}^{* 2}}\right]+\sigma_{h n f} B_{0}^{2} w_{1}^{* 2}+\phi^{*}
$$

where

$$
\phi^{*}=\mu_{h n f}\left[2\left(\frac{\partial u_{1}^{*}}{\partial r_{1}^{*}}\right)^{2}+\left(\frac{\partial u_{1}^{*}}{\partial z_{1}^{*}}+\frac{\partial w_{1}^{*}}{\partial r_{1}^{*}}\right)^{2}+2\left(\frac{\partial w_{1}^{*}}{\partial z_{1}^{*}}\right)^{2}\right]
$$


The equations for the thermophysical parameters of unitary nanofluid and hybrid nanofluid are described as given in Table (1a).

The geometry is subject to the following boundary and initial conditions ([46],[47]):

$$
\begin{gathered}
\left.w_{1}^{*}\left(r_{1}^{*}, t_{1}^{*}\right)\right|_{1} ^{*=R}=0,\left.\quad \frac{\partial w_{1}^{*}\left(r_{1}^{*}, t_{1}^{*}\right)}{\partial r_{1}^{*}}\right|_{r_{1}^{*}=0}=0, \quad w_{1}^{*}\left(r_{1}^{*}, 0\right)=0, \\
\left.\tilde{T}^{*}\left(r_{1}^{*}, t_{1}^{*}\right)\right|_{r_{1}^{*}=R}=\tilde{T}_{w}^{*},\left.\quad \frac{\partial \tilde{T}^{*}\left(r_{1}^{*}, t_{1}^{*}\right)}{\partial r_{1}^{*}}\right|_{r_{1}^{*}=0}=0, \quad \tilde{T}^{*}\left(r_{1}^{*}, 0\right)=0 .
\end{gathered}
$$

The first condition in Equation (7) indicates that there is no slip at the wall, the second is imposed due to the symmetry of the flow concerning the central plane, and the third suggests that the flow starts from the rest. The three temperature criteria in Equation (8) indicate the stated wall temperature, symmetry of temperature concerning the central plane, and a constant value of temperature inside the artery before the start of the flow.

Here, the temperature-dependent viscosity with Reynolds viscosity model [48] is considered, which is given as:

$$
\mu_{f}(\tilde{\theta})=\mu_{0} e^{-\beta_{0} \tilde{\theta}}=\mu_{0}\left[1-\beta_{0} \tilde{\theta}\right] \text { where } \beta_{0}<<1 \text {. }
$$

\subsection{Non-dimensionalization}

To achieve a numerical solution, the preceding governing Equations (2)-(5) are non-dimensionalized by introducing the following transformation variables ([46]):

$$
\begin{gathered}
\bar{r}_{1}^{*}=\frac{r_{1}^{*}}{R_{0}}, \bar{w}_{1}^{*}=\frac{w_{1}^{*}}{U_{0}}, \bar{u}_{1}^{*}=\frac{l_{0} u_{1}^{*}}{\delta^{*} U_{0}}, \bar{t}_{1}^{*}=\frac{U_{0} t_{1}^{*}}{R_{0}}, \bar{z}_{1}^{*}=\frac{z_{1}^{*}}{l_{0}}, \bar{p}_{1}^{*}=\frac{R_{0}^{2} p_{1}^{*}}{U_{0} l_{0} \mu_{0}}, \tilde{\theta}=\frac{\tilde{T}^{*}-\tilde{T}_{1}^{*}}{\tilde{T}_{w}^{*}-\tilde{T}_{1}^{*}}, \bar{R}=\frac{R}{R_{0}}, \bar{d}=\frac{d}{l_{0}}, \\
\operatorname{Re}=\frac{U_{0} \rho_{f} R_{0}}{\mu_{f}}, M^{2}=\frac{\sigma_{f} B_{0}^{2} R_{0}^{2}}{\mu_{f}}, G r=\frac{\rho_{f} R_{0}^{2} g \gamma_{f}\left(\tilde{T}_{w}^{*}-\tilde{T}_{1}^{*}\right)}{\mu_{f} U_{0}}, D a^{-1}=\frac{R_{0}^{2}}{k_{1}^{*}}, E c=\frac{U_{0}^{2}}{C_{p}\left(\tilde{T}_{w}^{*}-\tilde{T}_{1}^{*}\right)}, \operatorname{Pr}=\frac{\mu_{f} C_{p}}{k_{f}} .
\end{gathered}
$$




\begin{tabular}{|c|c|}
\hline Properties & Mathematical expression for nanofluid and hybrid nanofluid \\
\hline Viscosity & $\begin{array}{l}\mu_{n f}=\frac{\mu_{f}}{\left(1-\phi_{1}\right)^{2.5}} \\
\mu_{h n f}=\frac{\mu_{n} f}{\left(1-\phi_{1}\right)^{2.5}\left(1-\phi_{2}\right)^{2.5}}\end{array}$ \\
\hline Density & $\begin{array}{l}\rho_{n f}=\left(1-\phi_{1}\right) \rho_{f}+\phi_{1} \rho_{s_{1}} \\
\rho_{h n f}=\left[\left(1-\phi_{2}\right)\left\{\left(1-\phi_{1}\right) \rho_{f}+\phi_{1} \rho_{s_{1}}\right\}\right]+\phi_{2} \rho_{s_{2}}\end{array}$ \\
\hline Heat Capacity & $\begin{array}{l}\left(\rho C_{p}\right)_{n f}=\left(1-\phi_{1}\right)\left(\rho C_{p}\right)_{f}+\phi_{1}\left(\rho C_{p}\right)_{s_{1}} \\
\left(\rho C_{p}\right)_{h n f}=\left[\left(1-\phi_{2}\right)\left\{\left(1-\phi_{1}\right)\left(\rho C_{p}\right)_{f}+\phi_{1}\left(\rho C_{p}\right)_{s_{1}}\right\}\right]\end{array}$ \\
\hline Thermal Conductivity & $\begin{array}{l}\frac{k_{n f}}{k_{f}}=\frac{k_{s_{1}}+2 k_{f}-2 \phi_{1}\left(k_{f}-k_{s_{1}}\right)}{k_{s_{1}}+2 k_{f}+\phi_{1}\left(k_{f}-k_{s_{1}}\right)} \\
\frac{k_{h n f}}{k_{b f}}=\frac{k_{s_{2}}+2 k_{f}-2 \phi_{2}\left(k_{f}-k_{s_{2}}\right)}{k_{s_{2}}+2 k_{f}+\phi_{2}\left(k_{f}-k_{s_{2}}\right)} \text { where } \frac{k_{b f}}{k_{f}}=\frac{k_{s_{1}}+2 k_{f}-2 \phi_{1}\left(k_{f}-k_{s_{1}}\right)}{k_{s_{1}}+2 k_{f}+\phi_{1}\left(k_{f}-k_{s_{1}}\right)}\end{array}$ \\
\hline Electrical Conductivity & $\begin{array}{l}\frac{\sigma_{n f}}{\sigma_{f}}=\frac{\sigma_{s_{1}}+2 \sigma_{f}-2 \phi_{1}\left(\sigma_{f}-\sigma_{s_{1}}\right)}{\sigma_{s_{1}}+2 \sigma_{f}+\phi_{1}\left(\sigma_{f}-\sigma_{s_{1}}\right)} \\
\frac{\sigma_{h n f}}{\sigma_{b f}}=\frac{\sigma_{s_{2}}+2 \sigma_{f}-2 \phi_{2}\left(\sigma_{f}-\sigma_{s_{2}}\right)}{\sigma_{s_{2}}+2 \sigma_{f}+\phi_{2}\left(\sigma_{f}-\sigma_{s_{2}}\right)} \text { where } \frac{\sigma_{b f}}{\sigma_{f}}=\frac{\sigma_{s_{1}}+2 \sigma_{f}-2 \phi_{1}\left(\sigma_{f}-\sigma_{s_{1}}\right)}{\sigma_{s_{1}}+2 \sigma_{f}+\phi_{1}\left(\sigma_{f}-\sigma_{s_{1}}\right)}\end{array}$ \\
\hline Thermal Expansion Coefficient & $\begin{array}{l}\gamma_{n f}=\left(1-\phi_{1}\right) \gamma_{f}+\phi_{1} \gamma_{s_{1}} \\
\gamma_{h n f}=\left[\left(1-\phi_{2}\right)\left\{\left(1-\phi_{1}\right) \gamma_{f}+\phi_{1} \gamma_{s_{1}}\right\}\right]+\phi_{2} \gamma_{s_{2}}\end{array}$ \\
\hline
\end{tabular}

(a)

$\begin{array}{lll}\text { Parameters } & \text { Range } & \text { References } \\ \text { Hartmann Number }\left(M^{2}\right) & 0-5 & {[45],[6]} \\ \text { Grashof Number }(G r) & 0-0.5 & {[46],[7]} \\ \text { Inverse Darcy Number }\left(D a^{-1}\right) & 0.1-3 & {[47]} \\ \text { Reynold's Number }(R e) & 1-10 & {[46]} \\ \text { Eckert Number }(E c) & 0.1-1 & {[49]} \\ \text { Prandtl Number }(P r) & 14-25 & {[45],[4]}\end{array}$

(b)

\begin{tabular}{|ll} 
Parameters & Value \\
$\phi_{1}$ & 0.03 \\
$\phi_{2}$ & 0.03 \\
$B_{1}$ & 1.41 \\
$c_{1}$ & 1 \\
$\mathrm{e}$ & 0.2 \\
$\delta$ & 0.1 \\
$\beta_{0}$ & 0.5 \\
\hline
\end{tabular}

(c)

$\begin{array}{lccc}\text { Thermophysical Properties } & \text { Blood } & \text { Gold } & \text { Alumina } \\ \text { Density }\left[\rho\left(\mathrm{kg} / \mathrm{m}^{3}\right)\right] & 1063 & 19320 & 3970 \\ \text { Thermal Conductivity }[\mathrm{K}(\mathrm{W} / \mathrm{mK})] & 0.492 & 314 & 40 \\ \text { Electrical Conductivity }[\sigma(\mathrm{S} / \mathrm{m})] & 0.667 & 4.10 \times 10^{7} & 3.5 \times 10^{7} \\ \text { Thermal Expansion Coefficient }\left[\gamma \times 10^{-5}\left(\mathrm{~K}^{-1}\right)\right] & 0.18 & 1.4 & 0.85 \\ \text { Heat Capacitance }\left[C_{p}(\mathrm{~J} / \mathrm{kgK})\right] & 3594 & 129 & 765\end{array}$

(d)

Table 1: (a) Thermophysical parameters of nanofluid and hybrid nanofluid, (b) Range for various dimensionless parameters, (c) Default Values of emerging parameters, (d) Thermophysical properties of nanoparticles. 
After incorporating the above defined variables and neglecting the bars, the non-dimensionalized form of governing equations (2)-(5) are:

Continuity Equation:

$$
\delta\left[\frac{\partial u_{1}^{*}}{\partial r_{1}^{*}}+\frac{u_{1}^{*}}{r_{1}^{*}}\right]+\frac{\partial w_{1}^{*}}{\partial z_{1}^{*}}=0,
$$

\section{Momentum Equation:}

r-direction:

$$
\begin{aligned}
\operatorname{Re} \frac{\rho_{h n f}}{\rho_{f}} \delta \epsilon^{2}\left[\frac{\partial u_{1}^{*}}{\partial t_{1}^{*}}+(\delta \epsilon) u_{1}^{*}\right. & \left.\frac{\partial u_{1}^{*}}{\partial r_{1}^{*}}+\epsilon w_{1}^{*} \frac{\partial u_{1}^{*}}{\partial z_{1}^{*}}\right]=-\frac{\partial p_{1}^{*}}{\partial r_{1}^{*}}+\frac{\delta R_{0}}{l_{0}^{2}} \frac{1}{r_{1}^{*}} \frac{\partial}{\partial r_{1}^{*}}\left[\frac{\mu_{h n f}(\tilde{\theta})}{\mu_{0}} \frac{\partial u_{1}^{*}}{\partial r_{1}^{*}}\right] \\
& +\epsilon^{2} \frac{\partial}{\partial z_{1}^{*}}\left[\frac{\mu_{h n f}(\tilde{\theta})}{\mu_{0}}\left(\frac{\delta \epsilon}{l_{0}} \frac{\partial u_{1}^{*}}{\partial z_{1}^{*}}+\frac{1}{R_{0}} \frac{\partial w_{1}^{*}}{\partial r_{1}^{*}}\right)\right]-2 \delta \epsilon^{2} \frac{\mu_{h n f}(\tilde{\theta})}{\mu_{0}} \frac{u_{1}^{*}}{r_{1}^{* 2}}
\end{aligned}
$$

\section{z-direction:}

$$
\begin{aligned}
\operatorname{Re} \frac{\rho_{h n f}}{\rho_{f}}\left[\frac{\partial w_{1}^{*}}{\partial t_{1}^{*}}+\right. & \left.\delta \epsilon u_{1}^{*} \frac{\partial w_{1}^{*}}{\partial r_{1}^{*}}+\epsilon w_{1}^{*} \frac{\partial w_{1}^{*}}{\partial z_{1}^{*}}\right]=-\frac{\partial p_{1}^{*}}{\partial z_{1}^{*}}+\frac{1}{r_{1}^{*}} \frac{\partial}{\partial r_{1}^{*}}\left[r_{1}^{*} \frac{\mu_{h n f}(\tilde{\theta})}{\mu_{0}}\left(\delta \epsilon^{2} \frac{\partial u_{1}^{*}}{\partial z_{1}^{*}}+\frac{\partial w_{1}^{*}}{\partial r_{1}^{*}}\right)\right] \\
& +\epsilon^{2} \frac{\partial}{\partial z_{1}^{*}}\left[2 \frac{\mu_{h n f}(\tilde{\theta})}{\mu_{0}} \frac{\partial w_{1}^{*}}{\partial z_{1}^{*}}\right]+\frac{(\rho \gamma)_{h n f}}{(\rho \gamma)_{f}} G r \tilde{\theta}-\frac{\sigma_{h n f}}{\sigma_{f}} M^{2} w_{1}^{*}-\frac{\mu_{h n f}}{\mu_{0}} D a^{-1} w_{1}^{*}
\end{aligned}
$$

\section{Energy Equation:}

$$
\begin{aligned}
\frac{\left(\rho C_{p}\right)_{h n f}}{\left(\rho C_{p}\right)_{f}}\left[\frac{\partial \tilde{\theta}}{\partial t_{1}^{*}}+\delta \epsilon u_{1}^{*}\right. & \left.\frac{\partial \tilde{\theta}}{\partial r_{1}^{*}}+\epsilon w \frac{\partial \tilde{\theta}}{\partial z_{1}^{*}}\right]=\frac{1}{\operatorname{RePr}} \frac{k_{h n f}}{k_{f}}\left[\frac{\partial^{2} \tilde{\theta}}{\partial r_{1}^{* 2}}+\frac{1}{r_{1}^{*}} \frac{\partial \tilde{\theta}}{\partial r_{1}^{*}}+\epsilon^{2} \frac{\partial^{2} \tilde{\theta}}{\partial z_{1}^{* 2}}\right]+\frac{\sigma_{h n f}}{\sigma_{f}} \frac{E c M^{2}}{R e} w_{1}^{* 2} \\
+ & \frac{\mu_{h n f}}{\mu_{0}} \frac{E c}{\operatorname{Re}}\left[2 \delta^{2} \epsilon^{2}\left(\frac{\partial u_{1}^{*}}{\partial r_{1}^{*}}\right)^{2}+\left(\delta \epsilon^{2} \frac{\partial u_{1}^{*}}{\partial z_{1}^{*}}+\frac{\partial w_{1}^{*}}{\partial r_{1}^{*}}\right)^{2}+2 \epsilon^{2}\left(\frac{\partial w_{1}^{*}}{\partial z_{1}^{*}}\right)^{2}\right]
\end{aligned}
$$

The geometric parameters $\delta$ and $\epsilon$ in the above non-dimensionalized Equations (11)-(14) are stenosis height parameter and vessel aspect ratio, respectively. The mathematical expression representing them is $\delta=\delta^{*} / R_{0}$ and $\epsilon=R_{0} / l_{0}$.

The non-dimensional form of geometry of stenosis is: 


$$
R(z)= \begin{cases}1-\delta^{*} \operatorname{Exp}\left(-m^{2}\left(z-\frac{3}{2}\right)^{2}\right) \quad d \leq z \leq d+1 \\ 1 & \text { otherwise }\end{cases}
$$

Two further assumptions are implemented for the modification of the non-dimensionalized Equations (11)-(14): The maximum height of stenosis is lesser as compared with the radius of the artery i.e. $\delta<<1$, and the magnitude of the radius of the artery and the length of the stenotic region are comparable i.e. $\epsilon=O(1)$.

After implying the above made assumptions, the non-dimensionalized Equations (11)-(14) reduce to the following Equations (16)-(19):

\section{Continuity Equation:}

$$
\frac{\partial w_{1}^{*}}{\partial z_{1}^{*}}=0
$$

\section{Momentum Equation:}

\section{r-direction:}

$$
\frac{\partial p_{1}^{*}}{\partial r_{1}^{*}}=0
$$

\section{z-direction:}

$\operatorname{Re} \frac{\rho_{h n f}}{\rho_{f}} \frac{\partial w_{1}^{*}}{\partial t_{1}^{*}}=-\frac{\partial p_{1}^{*}}{\partial z_{1}^{*}}+\frac{1}{r_{1}^{*}} \frac{\partial}{\partial r_{1}^{*}}\left[r \frac{\mu_{h n f}(\tilde{\theta})}{\mu_{0}}\left(\frac{\partial w_{1}^{*}}{\partial r}\right)\right]+\frac{(\rho \gamma)_{h n f}}{(\rho \gamma)_{f}} G r \tilde{\theta}-\frac{\sigma_{h n f}}{\sigma_{f}} M^{2} w_{1}^{*}-\frac{\mu_{h n f}}{\mu_{0}} D a^{-1} w_{1}^{*}$,

\section{Energy Equation:}

$$
\frac{\left(\rho C_{p}\right)_{h n f}}{\left(\rho C_{p}\right)_{f}} \frac{\partial \tilde{\theta}}{\partial t_{1}^{*}}=\frac{1}{\operatorname{RePr}} \frac{k_{h n f}}{k_{f}}\left[\frac{\partial^{2} \tilde{\theta}}{\partial r_{1}^{* 2}}+\frac{1}{r_{1}^{*}} \frac{\partial \tilde{\theta}}{\partial r_{1}^{*}}\right]+\frac{\sigma_{h n f}}{\sigma_{f}} \frac{E c M^{2}}{\operatorname{Re}} w_{1}^{* 2}+\frac{\mu_{h n f}}{\mu_{0}} \frac{E c}{\operatorname{Re}}\left[\left(\frac{\partial w_{1}^{*}}{\partial r_{1}^{*}}\right)^{2}\right] .
$$

The axial pressure gradient can be expressed as follows [50]:

$$
-\frac{\partial p_{1}^{*}}{\partial z_{1}^{*}}=A_{0}+A_{1} \cos \left(2 \pi w_{p}\right) t_{1}^{*}, t_{1}^{*}>0
$$

where $A_{0}$ represents the mean pressure gradient and $A_{1}$ represents the amplitude of the pulsatile 
component that controls systolic and diastolic pressures.

The normalized form of the pressure gradient after substituting the non-dimensionalized variables becomes:

$$
-\frac{\partial p_{1}^{*}}{\partial z_{1}^{*}}=B_{1}\left[1+e \cos \left(c_{1} t_{1}^{*}\right)\right]
$$

where

$$
e=\frac{A_{1}}{A_{0}}, B_{1}=\frac{A_{0} R_{0}^{2}}{\mu_{0} U_{0}}, c_{1}=\frac{2 \pi R_{0} w_{p}}{U_{0}}
$$

Using Equation (21) in Equation (18), we have:

$$
\begin{array}{r}
\frac{\rho_{h n f}}{\rho_{f}} \operatorname{Re} \frac{\partial w_{1}^{*}}{\partial t_{1}^{*}}=B_{1}\left[1+e \cos \left(c_{1} t_{1}^{*}\right)\right]+\frac{1}{r_{1}^{*}} \frac{\partial}{\partial r_{1}^{*}}\left[r_{1}^{*} \frac{\mu_{h n f}(\tilde{\theta})}{\mu_{0}}\left(\frac{\partial w_{1}^{*}}{\partial r_{1}^{*}}\right)\right]+\frac{(\rho \gamma)_{h n f}}{(\rho \gamma)_{f}} G r \tilde{\theta}-\frac{\sigma_{h n f}}{\sigma_{f}} M^{2} w_{1}^{*} \\
-\frac{\mu_{h n f}}{\mu_{0}} D a^{-1} w_{1}^{*} \cdot
\end{array}
$$

The Equations (19) and (23) are subject to following boundary and initial conditions:

$$
\begin{gathered}
\left.w_{1}^{*}\left(r_{1}^{*}, t_{1}^{*}\right)\right|_{r_{1}^{*}=R}=0,\left.\frac{\partial w_{1}^{*}\left(r_{1}^{*}, t_{1}^{*}\right)}{\partial r_{1}^{*}}\right|_{r_{1}^{*}=0}=0, w_{1}^{*}\left(r_{1}^{*}, 0\right)=0, \\
\left.\tilde{\theta}\left(r_{1}^{*}, t_{1}^{*}\right)\right|_{r_{1}^{*}=R}=1,\left.\frac{\partial \tilde{\theta}\left(r_{1}^{*}, t_{1}^{*}\right)}{\partial r_{1}^{*}}\right|_{r_{1}^{*}=0}=0, \tilde{\theta}\left(r_{1}^{*}, 0\right)=0 .
\end{gathered}
$$

The wall shear stress (WSS), volumetric flow rate, resistance impedance, and heat transfer coefficient can be expressed mathematically as follows:

$$
\begin{gathered}
\tau_{w}=-\left(\frac{\partial w_{1}^{*}}{\partial r_{1}^{*}}\right)_{r_{1}^{*}=R}, \\
Q=2 \pi \int_{0}^{2 \pi} w_{1}^{*} r_{1}^{*} d r_{1}^{*},
\end{gathered}
$$




$$
\begin{gathered}
\lambda=\frac{L\left(\frac{\partial p_{1}^{*}}{\partial z_{1}^{*}}\right)}{Q}, \\
q_{w}=\left(\frac{\partial T}{\partial r_{1}^{*}}\right)_{r_{1}^{*}=R} .
\end{gathered}
$$

\subsection{Radial Coordinate Transformation}

To apply the computational scheme, the physical geometry is transformed into a rectangular domain using a suitable coordinate transformation given by $\left(x_{1}^{*}=\frac{r_{1}^{*}}{R\left(z_{1}^{*}\right)}\right)$

$$
\begin{array}{r}
\operatorname{Re} \frac{\rho_{h n f}}{\rho_{f}} \frac{\partial w_{1}^{*}}{\partial t_{1}^{*}}=B_{1}\left[1+e \cos \left(c_{1} t_{1}^{*}\right)\right]+\left(\frac{\mu_{h n f}(\tilde{\theta})}{\mu_{0}}\right)\left(\frac{1}{R_{1}^{* 2}}\right)\left[\frac{\partial^{2} w_{1}^{*}}{\partial x_{1}^{* 2}}+\frac{1}{x_{1}^{*}} \frac{\partial w_{1}^{*}}{\partial x_{1}^{*}}\right]+\frac{(\rho \gamma)_{h n f}}{(\rho \gamma)_{f}} G r \tilde{\theta} \\
-\frac{\sigma_{h n f}}{\sigma_{f}} M^{2} w_{1}^{*}-\frac{\mu_{h n f}}{\mu_{0}} D a^{-1} w_{1}^{*},
\end{array}
$$

$\frac{\left(\rho C_{p}\right)_{h n f}}{\left(\rho C_{p}\right)_{f}} \frac{\partial \tilde{\theta}}{\partial t_{1}^{*}}=\frac{1}{\operatorname{RePr}} \frac{k_{h n f}}{k_{f}}\left(\frac{1}{R^{2}}\right)\left[\frac{\partial^{2} \tilde{\theta}}{\partial x_{1}^{* 2}}+\frac{1}{x_{1}^{*}} \frac{\partial \tilde{\theta}}{\partial x_{1}^{*}}\right]+\frac{\sigma_{h n f}}{\sigma_{f}} \frac{E c M^{2}}{\operatorname{Re}} w_{1}^{* 2}+\frac{\mu_{h n f}}{\mu_{0}} \frac{E c}{\operatorname{Re}}\left(\frac{1}{R^{2}}\right)\left(\frac{\partial w_{1}^{*}}{\partial x_{1}^{*}}\right)^{2}$.

The associated boundary and initial conditions (24) and (25) becomes:

$$
\begin{gathered}
\left.\frac{\partial w_{1}^{*}}{\partial x_{1}^{*}}\right|_{x_{1}^{*}=0}=0,\left.w_{1}^{*}\right|_{x_{1}^{*}=1}=0,\left.\frac{\partial \tilde{\theta}}{\partial x_{1}^{*}}\right|_{x_{1}^{*}=0}=0,\left.\tilde{\theta}\right|_{x_{1}^{*}=1}=1, \\
\left.w_{1}^{*}\right|_{t_{1}^{*}=0}=0,\left.\tilde{\theta}\right|_{t_{1}^{*}=0}=0 .
\end{gathered}
$$

The wall shear stress (WSS), volumetric flow rate, resistance impedance, and heat transfer coefficient are expressed as: 


$$
\begin{gathered}
\tau_{w}=-\frac{1}{R}\left(\frac{\partial w_{1}^{*}}{\partial x_{1}^{*}}\right)_{x_{1}^{*}=1} \\
Q=2 \pi R^{2} \int_{0}^{2 \pi} w_{1}^{*} x_{1}^{*} d x_{1}^{*}, \\
\lambda=\frac{L\left(\frac{\partial p_{1}^{*}}{\partial z_{1}^{*}}\right)}{Q}, \\
q_{w}=\frac{1}{R}\left(\frac{\partial \tilde{\theta}}{\partial x_{1}^{*}}\right)_{x_{1}^{*}=1} .
\end{gathered}
$$

\section{Numerical Solution}

The partial differential equations (30) and (31) are coupled equations, therefore finding an exact solution for them is challenging. Hence, numerical methods are used for solving such coupled partial differential equations defined by (30) and (31). A reasonably advisable numerical scheme based on the finite difference method is Crank-Nicolson scheme, an implicit scheme. This method is well-known and has already been employed by several researchers ([45], [51]) in their studies. The Crank-Nicolson method is just an amalgamation of the Forward Euler and Backward Euler methods at $n^{\text {th }}$ and $(n+1)^{t h}$ levels, respectively. One of the main reasons for using this method is that it is unconditionally stable [52]. Further, it is second-order convergent both in time and space. Let us just ignore the subscripts and superscripts in Equations (30) and (31) and simply consider $w_{1}^{*}, x_{1}^{*}$ and $t_{1}^{*}$ as $w, x$ and $t$ for discretization. 


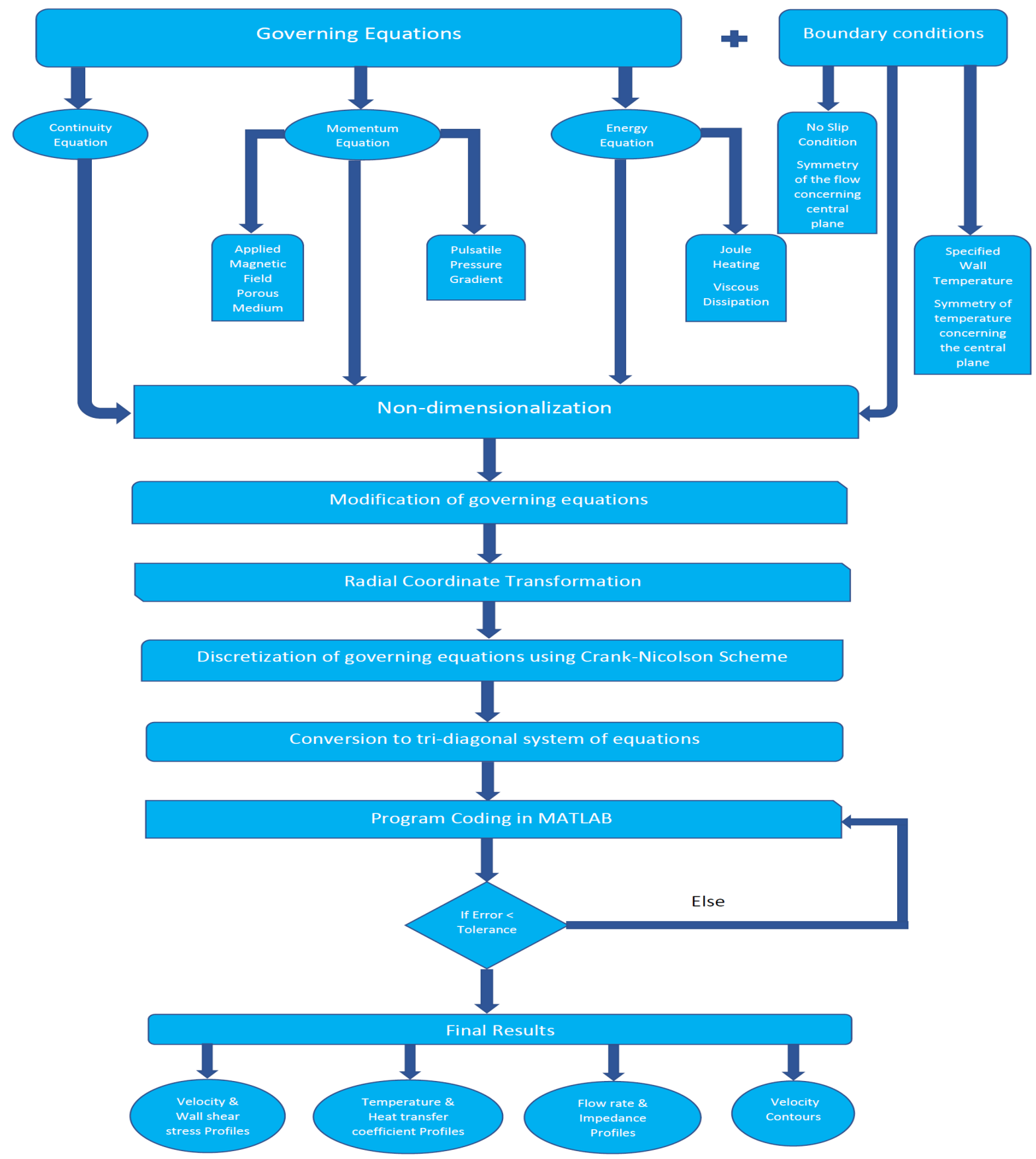

Figure 2: Schematic representation of the mathematical model using flow chart 


\subsection{Discretization of governing equations}

The discretization of the governing equations (30) and (31) is performed as follows:

$$
\begin{aligned}
& {\left[\left(1-\phi_{2}\right)\left[\left(1-\phi_{1}\right)+\phi_{1} \frac{\rho_{s 1}}{\rho_{f}}\right]+\phi_{2} \frac{\rho_{s 2}}{\rho_{f}}\right] \operatorname{Re}\left[\frac{w_{i}^{k+1}-w_{i}^{k}}{d t}\right]=B_{1}\left[1+e \cos \left(c_{1} t^{k}\right)\right]+} \\
& \frac{\left(1-\beta_{0} \tilde{\theta}_{i}^{k}\right)}{\left(1-\phi_{1}\right)^{2.5}\left(1-\phi_{2}\right)^{2.5}}\left(\frac{1}{R^{2}}\right)\left[\frac{1}{2}\left(\frac{w_{i+1}^{k+1}-2 w_{i}^{k+1}+w_{i-1}^{k+1}}{d x^{2}}+\frac{w_{i+1}^{k}-2 w_{i}^{k}+w_{i-1}^{k}}{d x^{2}}\right)\right. \\
& \left.+\frac{1}{2 x(i)}\left(\frac{w_{i+1}^{k+1}-w_{i-1}^{k+1}}{2 d x}+\frac{w_{i+1}^{k}-w_{i-1}^{k}}{2 d x}\right)\right]+\left[\left(1-\phi_{2}\right)\left[\left(1-\phi_{1}\right)+\phi_{1} \frac{(\rho \gamma)_{s 1}}{(\rho \gamma)_{f}}\right]+\phi_{2} \frac{(\rho \gamma)_{s 2}}{(\rho \gamma)_{f}}\right] G r \tilde{\theta}_{i}^{k} \\
& -\frac{1}{2} \frac{\sigma_{h n f}}{\sigma_{f}} M^{2}\left(w_{i}^{k}+w_{i}^{k+1}\right)-\frac{1}{2} \frac{\left(1-\beta_{0} \tilde{\theta}_{i}^{k}\right)}{\left(1-\phi_{1}\right)^{2.5}\left(1-\phi_{2}\right)^{2.5}} D a^{-1}\left(w_{i}^{k}+w_{i}^{k+1}\right), \\
& {\left[\left(1-\phi_{2}\right)\left[\left(1-\phi_{1}\right)+\phi_{1} \frac{\left(\rho C_{p}\right)_{s 1}}{\left(\rho C_{p}\right)_{f}}\right]+\phi_{2} \frac{\left(\rho C_{p}\right)_{s 2}}{\left(\rho C_{p}\right)_{f}}\right]\left[\frac{\tilde{\theta}_{i}^{k+1}-\tilde{\theta}_{i}^{k}}{d t}\right]} \\
& =\frac{1}{\operatorname{RePr}} \frac{k_{h n f}}{k_{f}}\left(\frac{1}{R^{2}}\right)\left[\frac{1}{2}\left(\frac{\tilde{\theta}_{i+1}^{k+1}-2 \tilde{\theta}_{i}^{k+1}+\tilde{\theta}_{i-1}^{k+1}}{d x^{2}}+\frac{\tilde{\theta}_{i+1}^{k}-2 \tilde{\theta}_{i}^{k}+\tilde{\theta}_{i-1}^{k}}{d x^{2}}\right)\right. \\
& \left.+\frac{1}{2 x(i)}\left(\frac{\tilde{\theta}_{i+1}^{k+1}-\tilde{\theta}_{i-1}^{k+1}}{2 d x}+\frac{\tilde{\theta}_{i+1}^{k}-\tilde{\theta}_{i-1}^{k}}{2 d x}\right)\right]+\frac{\sigma_{h n f}}{\sigma_{f}} \frac{E c M^{2}}{\operatorname{Re}}\left[\frac{1}{2}\left(w_{i}^{k+1}+w_{i}^{k}\right)\right]^{2}+ \\
& \frac{\left(1-\frac{\beta_{0}}{2}\left(\tilde{\theta}_{i}^{k}+\tilde{\theta}_{i}^{k+1}\right)\right)}{\left(1-\phi_{1}\right)^{2.5}\left(1-\phi_{2}\right)^{2.5}} \frac{E c}{R e}\left(\frac{1}{R^{2}}\right)\left[\frac{1}{2}\left(\frac{w_{i+1}^{k+1}-w_{i-1}^{k+1}}{2 d x}+\frac{w_{i+1}^{k}-w_{i-1}^{k}}{2 d x}\right)\right]^{2} .
\end{aligned}
$$

The associated boundary conditions are also discretized and they get reduced as follows:

$$
\begin{aligned}
& w_{-1}^{k+1}+w_{-1}^{k}=w_{1}^{k+1}+w_{1}^{k}, \quad w_{N}^{k+1}=0, \\
& \tilde{\theta}_{-1}^{k+1}+\tilde{\theta}_{-1}^{k}=\tilde{\theta}_{1}^{k+1}+\tilde{\theta}_{1}^{k}, \quad \tilde{\theta}_{N}^{k+1}=1 .
\end{aligned}
$$

Here, the spatial variable is discretized into $\mathrm{N}+1$ grid points considering the step size as $\mathrm{x}=$ $1 / \mathrm{N}+1$. The time instant is determined by the value of $t^{k}$, which is given as $t^{k}=(k-1) d t$, where $d t$ denotes a small increment in time. Although the method is stable for all values of $d t$ and $d x$, the values have been chosen very precisely as $d t=0.0001$ and $d x=0.0001$. Also, no further 
change is noticed in the results with a decrement in these values. As mentioned earlier, the method is implicit one, therefore the governing Equations (38) and (39) constitute a tri-diagonal system of equations and can be solved easily using the Tri-diagonal Matrix Algorithm(TDMA) [53].

Equation (38) corresponds to a tri-diagonal system, which is given by

$$
A_{i}^{k} w_{i-1}^{k+1}+B_{i}^{k} w_{i}^{k+1}+C_{i}^{k} w_{i+1}^{k+1}=A_{i}^{\prime k} w_{i-1}^{k}+B_{i}^{\prime k} w_{i}^{k}+C_{i}^{\prime k} w_{i+1}^{k}+D_{i}^{k}
$$

where

$$
\begin{aligned}
& A_{i}^{k}=-\frac{\left(1-\beta_{0} \tilde{\theta}_{i}^{k}\right)}{\left(1-\phi_{1}\right)^{2.5}\left(1-\phi_{2}\right)^{2.5}}\left(\frac{1}{2 R^{2}}\right)\left(\frac{d t}{d x^{2}}-\frac{1}{2 x(i)} \frac{d t}{d x}\right) \\
& B_{i}^{k}=\operatorname{Re}\left[\left(1-\phi_{2}\right)\left[\left(1-\phi_{1}\right)+\phi_{1} \frac{\rho_{s 1}}{\rho_{f}}\right]+\phi_{2} \frac{\rho_{s 2}}{\rho_{f}}\right]+\frac{\left(1-\beta_{0} \tilde{\theta}_{i}^{k}\right)}{\left(1-\phi_{1}\right)^{2.5}\left(1-\phi_{2}\right)^{2.5}} \frac{1}{R^{2}} \frac{d t}{d x^{2}}+\frac{d t}{2} \frac{\sigma_{h n f}}{\sigma_{f}} M^{2}+\frac{d t}{2} \frac{\left(1-\beta_{0} \tilde{\theta}_{i}^{k}\right)}{\left(1-\phi_{1}\right)^{2.5}\left(1-\phi_{2}\right)^{2.5}} D a^{-1} \\
& C_{i}^{k}=-\frac{\left(1-\beta_{0} \tilde{\theta}_{i}^{k}\right)}{\left(1-\phi_{1}\right)^{2.5}\left(1-\phi_{2}\right)^{2.5}}\left(\frac{1}{2 R^{2}}\right)\left(\frac{d t}{d x^{2}}+\frac{1}{2 x(i)} \frac{d t}{d x}\right) \\
& A_{i}^{\prime k}=\frac{\left(1-\beta_{0} \tilde{\theta}_{i}^{k}\right)}{\left(1-\phi_{1}\right)^{2.5}\left(1-\phi_{2}\right)^{2.5}}\left(\frac{1}{2 R^{2}}\right)\left(\frac{d t}{d x^{2}}-\frac{1}{2 x(i)} \frac{d t}{d x}\right) \\
& B_{i}^{\prime k}=\operatorname{Re}\left[\left(1-\phi_{2}\right)\left[\left(1-\phi_{1}\right)+\phi_{1} \frac{\rho_{s 1}}{\rho_{f}}\right]+\phi_{2} \frac{\rho_{s 2}}{\rho_{f}}\right]-\frac{\left(1-\beta_{0} \tilde{\theta}_{i}^{k}\right)}{\left(1-\phi_{1}\right)^{2.5}\left(1-\phi_{2}\right)^{2.5}} \frac{1}{R^{2}} \frac{d t}{d x^{2}}-\frac{d t}{2} \frac{\sigma_{h n f}}{\sigma_{f}} M^{2}-\frac{d t}{2} \frac{\left(1-\beta_{0} \tilde{\theta}_{i}^{k}\right)}{\left(1-\phi_{1}\right)^{2.5}\left(1-\phi_{2}\right)^{2.5}} D a^{-1} \\
& C_{i}^{\prime k}=\frac{\left(1-\beta_{0} \tilde{\theta}_{i}^{k}\right)}{\left(1-\phi_{1}\right)^{2.5}\left(1-\phi_{2}\right)^{2.5}}\left(\frac{1}{2 R^{2}}\right)\left(\frac{d t}{d x^{2}}+\frac{1}{2 x(i)} \frac{d t}{d x}\right) \\
& D_{i}^{k}=d t B_{1}\left[1+e \cos \left(c_{1} t^{k}\right)\right]+d t\left[\left(1-\phi_{2}\right)\left[\left(1-\phi_{1}\right)+\phi_{1} \frac{(\rho \gamma)_{s 1}}{(\rho \gamma)_{f}}\right]+\phi_{2} \frac{(\rho \gamma)_{s 2}}{(\rho \gamma)_{f}}\right] \operatorname{Gr} \tilde{\theta}_{i}^{k}
\end{aligned}
$$

The tri-diagonal system corresponding to Equation (39) is given by

$$
P_{i}^{k} \theta_{i-1}^{k+1}+Q_{i}^{k} \theta_{i}^{k+1}+S_{i}^{k} \theta_{i+1}^{k+1}=P_{i}^{\prime k} \theta_{i-1}^{k}+Q_{i}^{\prime k} \theta_{i}^{k}+S_{i}^{\prime k} \theta_{i+1}^{k}+F_{i}^{k}
$$

where $P_{i}^{k}=-\frac{1}{\operatorname{RePr}} \frac{k_{h n f}}{k_{f}}\left(\frac{1}{2 R^{2}}\right)\left(\frac{d t}{d x^{2}}-\frac{1}{2 x(i)} \frac{d t}{d x}\right)$

$$
\begin{gathered}
Q_{i}^{k}=\left[\left(1-\phi_{2}\right)\left[\left(1-\phi_{1}\right)+\phi_{1} \frac{\left(\rho C_{p}\right)_{s 1}}{\left(\rho C_{p}\right)_{f}}\right]+\phi_{2} \frac{\left(\rho C_{p}\right)_{s 2}}{\left(\rho C_{p}\right)_{f}}\right]+\frac{1}{\operatorname{RePr} \frac{k_{h n f}}{k_{f}} \frac{1}{R^{2}} \frac{d t}{d x^{2}}} \\
\quad+\frac{d t}{\left(1-\phi_{1}\right)^{2.5}\left(1-\phi_{2}\right)^{2.5}} \frac{\beta_{0}}{2} \frac{E c}{\operatorname{Re}}\left(\frac{1}{R^{2}}\right)\left[\frac{1}{2}\left(\frac{w_{i+1}^{k+1}-w_{i-1}^{k+1}}{2 d x}+\frac{w_{i+1}^{k}-w_{i-1}^{k}}{2 d x}\right)\right]^{2} \\
S_{i}^{k}=-\frac{1}{\operatorname{RePr}} \frac{k_{h n f}}{k_{f}}\left(\frac{1}{2 R^{2}}\right)\left(\frac{d t}{d x^{2}}+\frac{1}{2 x(i)} \frac{d t}{d x}\right)
\end{gathered}
$$




$$
\begin{aligned}
& P_{i}^{\prime k}= \frac{1}{\operatorname{RePr}} \frac{k_{h n f}}{k_{f}}\left(\frac{1}{2 R^{2}}\right)\left(\frac{d t}{d x^{2}}-\frac{1}{2 x(i)} \frac{d t}{d x}\right) \\
& Q_{i}^{\prime k}= {\left[\left(1-\phi_{2}\right)\left[\left(1-\phi_{1}\right)+\phi_{1} \frac{\left(\rho C_{p}\right)_{s 1}}{\left(\rho C_{p}\right)_{f}}\right]+\phi_{2} \frac{\left(\rho C_{p}\right)_{s 2}}{\left(\rho C_{p}\right)_{f}}\right]-\frac{1}{\operatorname{RePr} \frac{k_{h n f}}{k_{f}} \frac{1}{R^{2}} \frac{d t}{d x^{2}}} } \\
& \quad-\frac{d t}{\left(1-\phi_{1}\right)^{2.5}\left(1-\phi_{2}\right)^{2.5}} \frac{\beta_{0}}{2} \frac{E c}{R e}\left(\frac{1}{R^{2}}\right)\left[\frac{1}{2}\left(\frac{w_{i+1}^{k+1}-w_{i-1}^{k+1}}{2 d x}+\frac{w_{i+1}^{k}-w_{i-1}^{k}}{2 d x}\right)\right]^{2} \\
& S_{i}^{\prime k}=\frac{1}{\operatorname{RePr}} \frac{k_{h n f}}{k_{f}}\left(\frac{1}{2 R^{2}}\right)\left(\frac{d t}{d x^{2}}+\frac{1}{2 x(i)} \frac{d t}{d x}\right) \\
& F_{i}^{k}=d t \frac{\sigma_{h n f}}{\sigma_{f}} \frac{E c M^{2}}{\operatorname{Re}}\left[\frac{1}{2}\left(w_{i}^{k+1}+w_{i}^{k}\right)\right]^{2}+\frac{d t}{\left(1-\phi_{1}\right)^{2.5}\left(1-\phi_{2}\right)^{2.5}} \frac{\beta_{0}}{2} \frac{E c}{\operatorname{Re}}\left(\frac{1}{R^{2}}\right)\left[\frac{1}{2}\left(\frac{w_{i+1}^{k+1}-w_{i-1}^{k+1}}{2 d x}+\frac{w_{i+1}^{k}-w_{i-1}^{k}}{2 d x}\right)\right]^{2}
\end{aligned}
$$

\section{Results and Graphical Analysis}

This investigation aimed to see the behaviour of blood flow through an artery with stenosis under the influence of pulsatile blood flow subject to an external magnetic field. The influence of various dimensionless parameters such as Magnetic number $\left(M^{2}\right)$, Darcy number $(D a)$, Grashof number $(G r)$, viscosity parameter $\left(\beta_{0}\right)$, Reynolds number $(R e)$, Eckert Number $(E c)$, Prandtl number $(\operatorname{Pr})$, different concentration of both the nanoparticles $\left(\phi_{1}, \phi_{2}\right)$, and pressure gradient parameter $\left(B_{1}\right)$ has been examined graphically for velocity, temperature, wall shear stress, volumetric flow rate, impedance and heat transfer coefficient profiles. The velocity contours for different emerging parameters have been drawn to analyze the overall behavior of blood flow patterns. The range for various non-dimensional parameters is represented in Table (1b). The computational work has been carried out by using the data illustrated in the Table (1c) and (1d). The schematic representation of the work plan is depicted in Figure (2) with the aid of a flow chart.

\subsection{Validation of the numerical results}

The validation of the present finite difference numerical code is conducted by benchmarking with Tripathi's published work [46] for the Au-nanoparticles, which is common in both the research work. For authentication, the graphs (3a-3b) have been plotted for non-dimensional velocity and temperature profiles against the Grashof number $(G r=0.2)$ and Prandtl number $(\operatorname{Pr}=21)$, respectively. To compare both the research work, we have neglected Magnetic number $\left(M^{2}\right)$, Darcy 
number $(D a)$, and Eckert number $(E c)$. There is a good agreement between our results and [46].

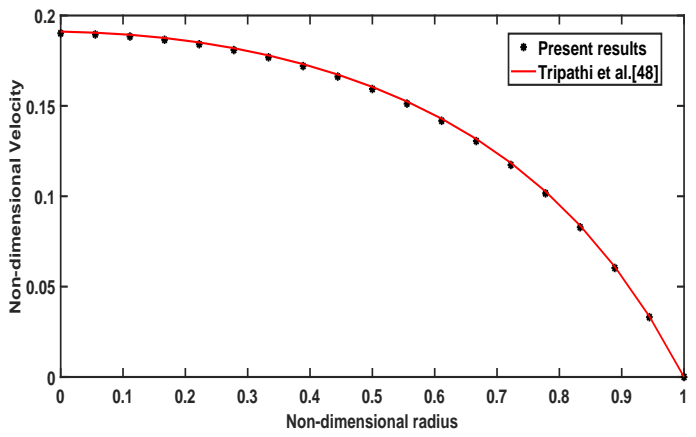

(a)

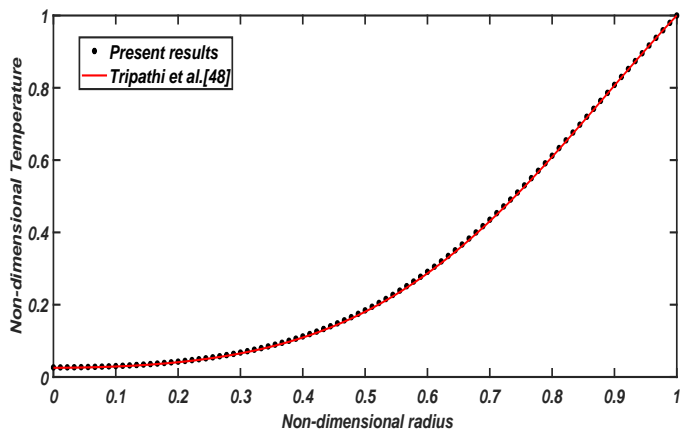

(b)

Figure 3: Comparison graphs for (a) non-dimensional velocity profile for Grashof Number $(G r=0.2)$, (b) non-dimensional temperature profile for Prandtl Number (Pr=21)

\subsection{Axial Velocity Profiles}

The effect of various flow parameters such as Magnetic Number $\left(M^{2}\right)$, Darcy Number $(D a)$, Grashof Number $(G r)$, viscosity parameter $\left(\beta_{0}\right)$, Reynolds Number $(R e)$, different concentrations of both nanoparticles $\left(\phi_{1}, \phi_{2}\right)$ and pressure gradient parameter $\left(B_{1}\right)$ on non-dimensional velocity profile is depicted in Figure (4). The non-dimensional velocity profile for $M^{2}$ is shown in Figure (4a). The profiles reveal that increasing the strength of the magnetic field causes a reduction in blood velocity. A stronger magnetic field boosts the Lorentzian magnetic drag force, producing greater deceleration in the blood flow. Lorentz force is generated when charged particles pass through a strong magnetic field fastly, counteracting blood motion. As a result, the magnetic field plays a critical role in blood flow dynamics in arteries. It is thus utilized as a valuable tool in treating various cardiovascular disorders. In MRI, static magnetic fields are utilized to reduce the time it takes to heal fractures and regenerate nerves. Comparing the profiles for Au-blood nanofluid and $\mathrm{Au}-\mathrm{Al}_{2} \mathrm{O}_{3}$ /blood hybrid nanofluid, it is analyzed that hybrid nanofluid effectively decreases blood velocity, which is very advantageous for blood flow regulation in arterial illnesses. The illustration of $D a$ is given in Figure (4b). With increasing $D a$, the velocity profile enhances, indicating that the medium's permeability provides less barrier to the flow, and hence the velocity rises. Darcy 


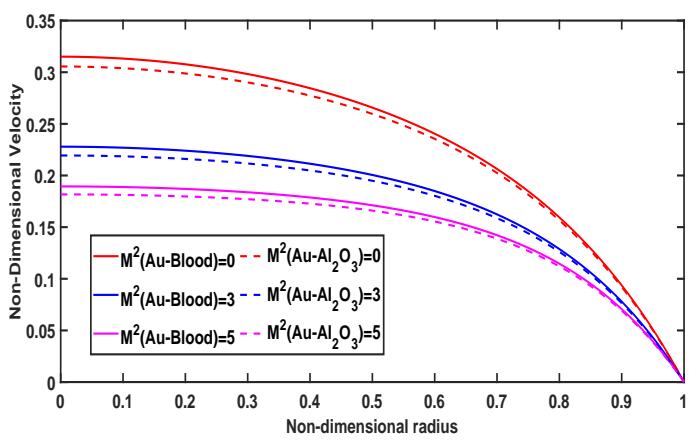

(a)

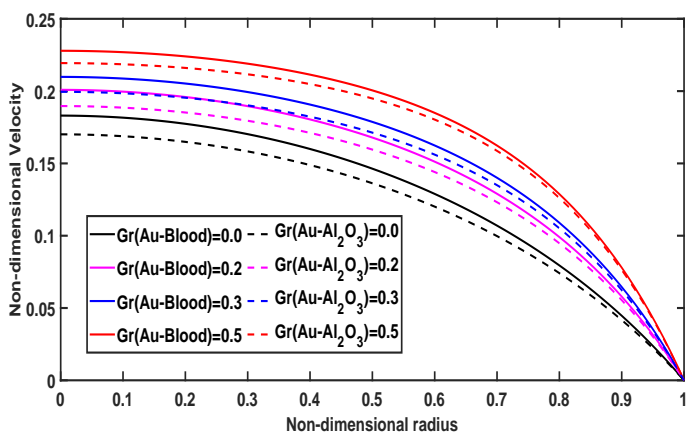

(c)

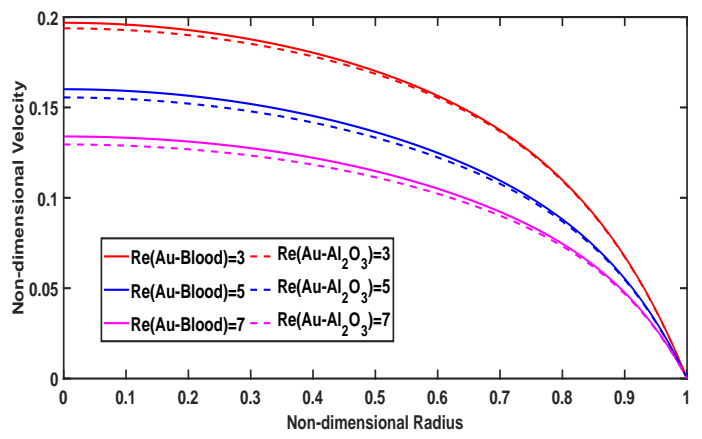

(e)

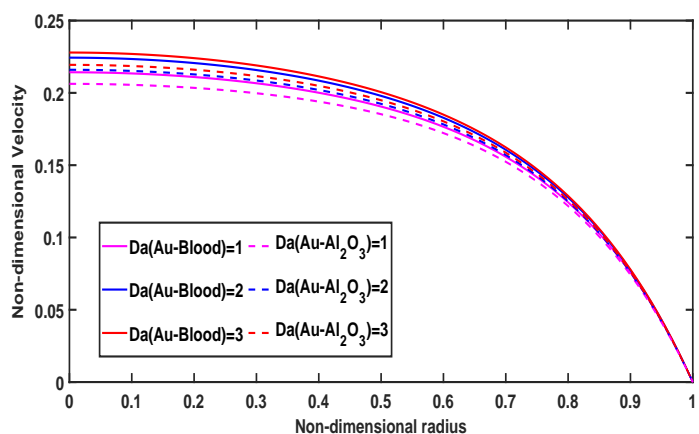

(b)

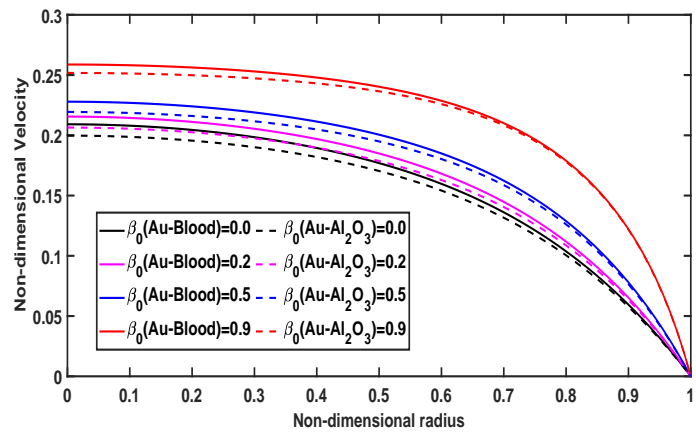

(d)

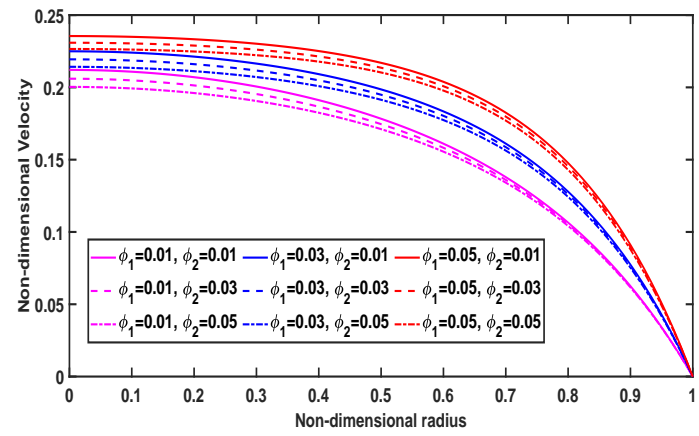

(f)

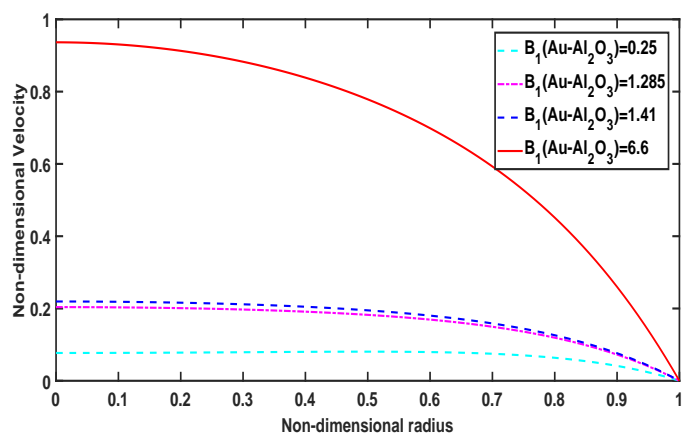

$(\mathrm{g})$

Figure 4: Influence of (a) Magnetic Number $\left(M^{2}\right)$, (b) Darcy Number ( $\left.D a\right)$, (c) Grashof Number $(G r)$, (d) viscosity parameter $\left(\beta_{0}\right)$, (e) Reynolds Number $(R e)$, (f) different concentrations of both nanoparticles $\left(\phi_{1}, \phi_{2}\right)$, $(\mathrm{g})$ pressure gradient parameter $\left(B_{1}\right)$ on non-dimensional velocity at $z_{1}^{*}=1.5$ and $t_{1}^{*}=1.3$ 
number is physically related to the permeability of a porous surface. The matrix resistance provided by solid fibers in the porous media is reduced as the Darcy number increases, increasing the non-dimensional blood velocity. Figure (4c) outlines the impact of $G r$ on the non-dimensional velocity profile. A rise in non-dimensional velocity is observed with increasing $G r$ values since the increase in $G r$ values results in a higher thermal buoyancy force and stronger thermal convection currents in the blood flow. As a result, thermal buoyancy significantly impacts hemodynamic momentum parameters. The maximum value of velocity is observed at the centerline of the artery in all the cases. Figure (4d) describes the effect of $\beta_{0}$ on the non-dimensional velocity profile. The parameter $\beta_{0}$ causes the blood flow velocity to accelerate. The increase in velocity indicates that the resistance among the particles has decreased as the viscosity parameter's magnitude has increased. With the enhancement in $\beta_{0}$, the fluid becomes less viscous, and therefore an acceleration in the flow velocity is witnessed. The temperature-dependent viscosity model is employed in this work as a variable viscosity model (Equation(9)). The variation of the non-dimensional velocity profile for different values of $R e$ is demonstrated in Figure (4e). A rise in $R e$ values from 3 to 7 results in a velocity declination since for low $R e$ values, the regime becomes viscous-dominated. Even though inertial force increases with Reynolds number, flow retardation is the dominant effect due to stenotic occlusion and the presence of nanoparticles. The Reynolds number is used to classify fluid systems in which the effect of viscosity is crucial in determining fluid velocities or flow patterns. The impact of nanoparticle concentration for $\mathrm{Au}-\mathrm{Al}_{2} \mathrm{O}_{3}$ /blood hybrid nanofluid on the non-dimensional velocity profile is illustrated in Figure (4f). The minimum value of velocity is depicted for $\phi_{1}=0.01, \phi_{2}=0.05$ and maximum value is observed for $\phi_{1}=0.05, \phi_{2}=0.01$ which shows that both the nanoparticles have opposite effect on velocity. An increment in the concentration of Au-nanoparticles increases the velocity, while a decrease in the concentration of $\mathrm{Al}_{2} \mathrm{O}_{3}$-nanoparticles enhances velocity. This gives the advantage of putting blood velocity under control and allows the surgeons to adjust it as and when needed. Figure (4g) displays the influence of $B_{1}$ on non-dimensional velocity profiles. The profile of axial blood velocity shows a hike with increasing values of $B_{1}$, as depicted in the Figure $(4 \mathrm{~g})$. In particular for $B_{1}=1.41$ represents coro- 
nary artery and $B_{1}=6.6$ for femoral artery as described in [54], at a specific position in the stenotic zone $(\mathrm{z}=1.5)$. The blood flow is accelerated as the pressure parameter is increased, demonstrating that the femoral artery reaches its maximum value and considerably exceeds the axial velocity computed for the coronary artery.

\subsection{Temperature Profiles}

The influence of different parameters such as Eckert Number $(E c)$, the combined effect of Joule heating $\left(M^{2}\right)$ and viscous dissipation $(E c)$, Prandtl Number (Pr), Reynolds Number (Re), and different concentrations of both nanoparticles $\left(\phi_{1}, \phi_{2}\right)$ on non-dimensional temperature profile is illustrated in Figure (5). Figure (5a) represents the effect of $E c$ on non-dimensional temperature profile. The increase in the $E c$ illustrates an increase in the temperature distribution in the flow region as frictional heating causes heat energy to build in the fluid. The temperature rises significantly due to viscous dissipation in the flow, which also causes an increase in the thickness of the thermal boundary layer. Physically, nanoparticles disperse energy in the form of heat, which raises the temperature. As a result, adding additional nanoparticles (e.g., hybrid nanofluid) can use more energy, improving the temperature and thickness of the boundary layer even more. Figure (5b) depicts the cumulative impact of Joule Heating and viscous dissipation. It demonstrates that increasing viscous dissipation $(E c)$ and Joule heating $\left(M^{2}\right)$ simultaneously raises the nanofluid temperature since the mechanical energy is transformed to thermal energy within molecules, which causes a hike in temperature. The profiles demonstrate a good agreement with Venkatesan's [49] findings. Joule heating, also known as Ohmic dissipation characterizes the supplementary destruction in mechanical energy with work done in dragging against the action of the magnetic field. This generates heat and boosts temperatures. The impact of $\operatorname{Pr}$ on non-dimensional temperature profiles is expressed by Figure (5c). The heat transmission from the artery diminishes when the Prandtl number increases as the $\operatorname{Pr}$ varies inversely with the heat transmission from the artery's wall to the fluid(blood), resulting in a decrease in arterial blood temperature. As a result, we may physically deduce that when Pr is small, heat diffuses relatively quickly compared to velocity. As 


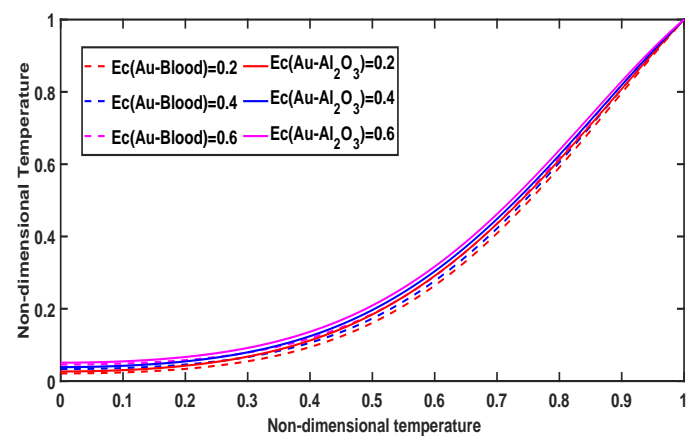

(a)

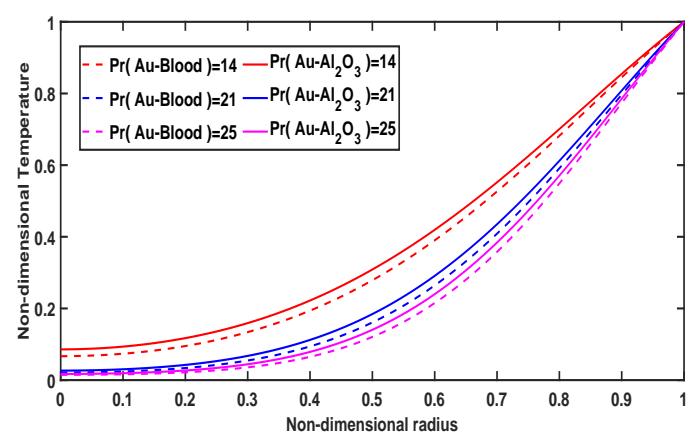

(c)

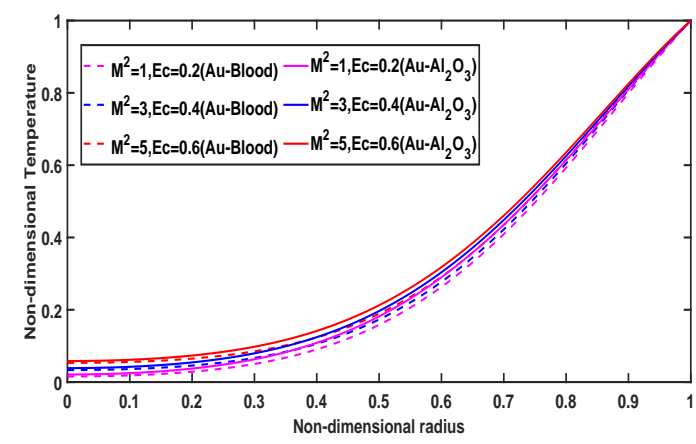

(b)

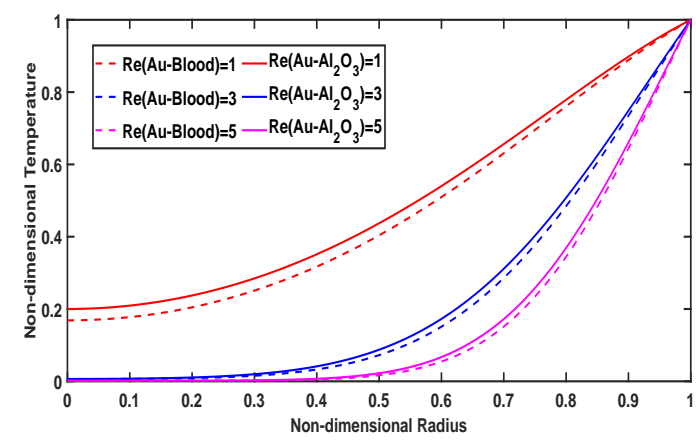

(d)

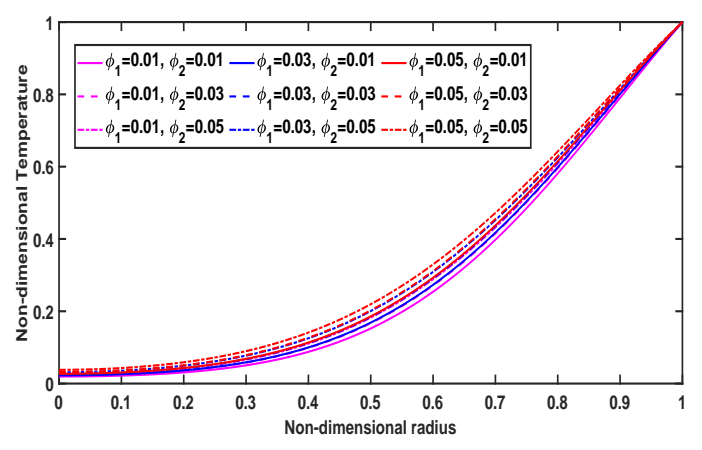

(e)

Figure 5: Influence of (a) Eckert Number $(E c)$, (b) combined effect of Joule heating $\left(M^{2}\right)$ and viscous dissipation $(E c)$, (c) Prandtl Number $(P r)$, (d) Reynolds Number $(R e)$, (e) different concentrations of both nanoparticles $\left(\phi_{1}, \phi_{2}\right)$ on non-dimensional temperature at $z_{1}^{*}=1.5$ and $t_{1}^{*}=1.3$

Pr rises, heat diffusibility decreases, allowing the temperature of blood flowing through the artery to increase. Figure (5d) portrays the non-dimensional temperature profile for various values of $R e$. A decrease in the temperature profile is observed with the increase in $R e$ values. It is the ratio of inertial forces to viscous forces, determined by the fluid's characteristic length, velocity, density, and viscosity. Low Reynolds numbers indicate that the viscous forces are more significant than in- 
ertial forces resulting in a decrement of the non-dimensional temperature profile. The influence of nanoparticle concentration on the non-dimensional temperature profile for $\mathrm{Au}-\mathrm{Al}_{2} \mathrm{O}_{3} /$ blood hybrid nanofluid is shown in Figure (5e). The minimum temperature value is for $\phi_{1}=0.01, \phi_{2}=0.01$, while the maximum value is for $\phi_{1}=0.05, \phi_{2}=0.05$, indicating that as the concentration of both nanoparticles falls, the temperature drops, which has the advantage of regulating temperature and allows to make adjustments as needed.

\subsection{Wall Shear Stress}

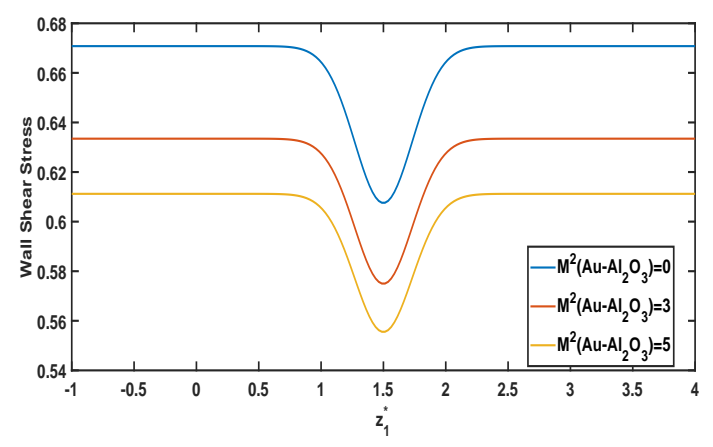

(a)

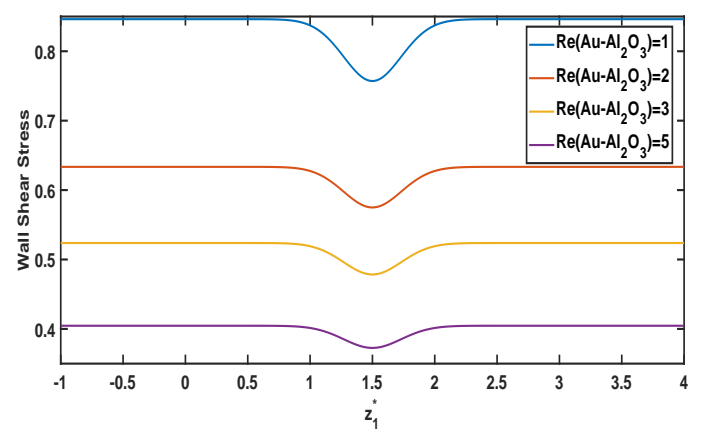

(c)

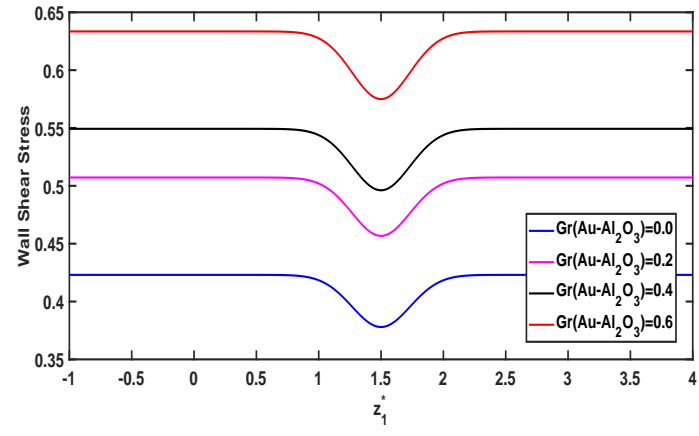

(b)

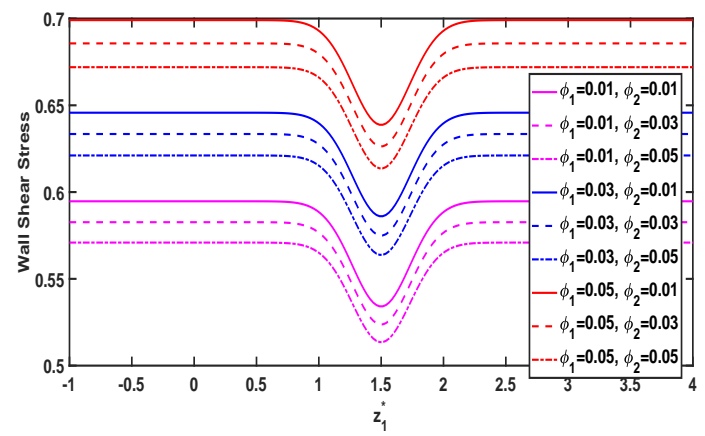

(d)

Figure 6: Effect of (a) Magnetic number $\left(M^{2}\right)$, (b) Grashof number $(G r)$, (c) Reynolds number $(R e)$, (d) different concentrations of both nanoparticles $\left(\phi_{1}, \phi_{2}\right)$ on non-dimensional velocity on Wall Shear Stress at $t_{1}^{*}=1.3$

In arterial blood flow, the force per unit area exerted on the fluid by the wall in a direction parallel to the local tangent plane is known as the wall shear stress. The atherogenic process is controlled by the pulsatile blood flow, which causes wall shear stress. According to recent 
research, low wall shear stress or oscillating wall shear stress vessel segments appear to be the most vulnerable to atherosclerotic plaques [55]. The wall shear stress decreases downstream of the stenotic zone, reaches a minimum at the stenosed region's midpoint $(\mathrm{z}=1.5)$, and increases upstream of the stenosis. Figure (6) represents wall shear stress profiles for different parameters such as Magnetic Number $\left(M^{2}\right)$, Grashof Number $(G r)$, Reynolds Number $(R e)$, and different nanoparticle concentration $\left(\phi_{1}, \phi_{2}\right)$. There is a reduction in wall shear stress as there is an increment in $M^{2}$ values (Figure(6b)) because an opposing force to the flow, known as the Lorentz force, is generated, resulting in decreased velocity and hence a decrease in wall shear stress. The wall shear stress profiles for $G r$ are represented in Figure(6b). The Grashof number $(G r)$ is the proportion of buoyancy to viscous force. The thermal buoyancy force is increased because nanoparticle species diffusion is impacted by nanoparticle concentration changes inside the blood. This phenomenon also causes a decrease in blood flow resistance and, as a result, an acceleration and, therefore, an increase in wall shear stress profiles. Figure (6c) marks the variation in wall shear stress profiles for different $R e$ values. Wall shear stress decreases with an increment in $R e$ values as low $R e$ values indicate that the viscous forces are more significant than inertial forces resulting in a decrement of the velocity and hence wall shear stress profiles. Therefore, the wall shear stress profiles get retarded due to reduced blood velocity. Figure (6d) shows the variation in wall shear stress for different nanoparticle concentrations. Increases in Au-nanoparticle concentration lead to a rise in wall shear stress, whereas increases in $\mathrm{Al}_{2} \mathrm{O}_{3}$-nanoparticles lead to a declination in wall shear stress profiles.

\subsection{Volumetric Flow Rate and Resistance Impedance}

The volumetric flow rate is the amount of fluid(blood) that flows in a given amount of time, while the ratio of pressure drop to flow rate is known as fluid resistance. Changes in resistance are the most common way for organs to regulate blood flow. Impedance and flow rate are inversely proportional. Flow rate and impedance profiles for different parameters such as Magnetic Number $\left(M^{2}\right)$, Grashof Number $(G r)$, Reynolds Number $(R e)$, and stenotic depth $(\delta)$, are displayed in Fig- 


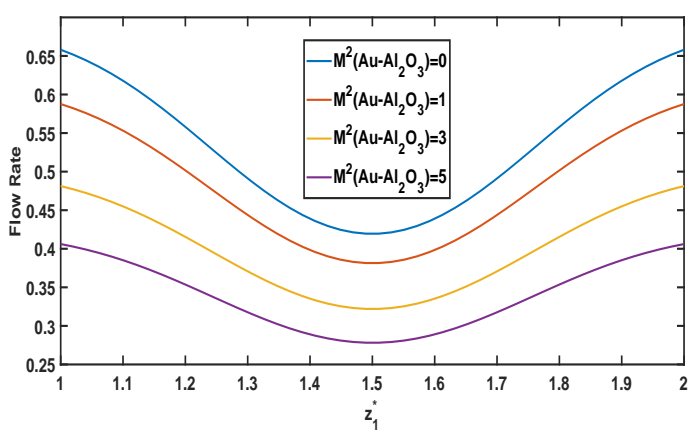

(a)

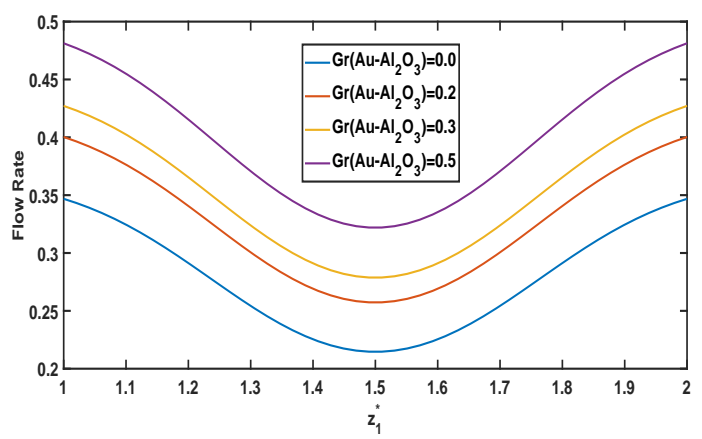

(c)

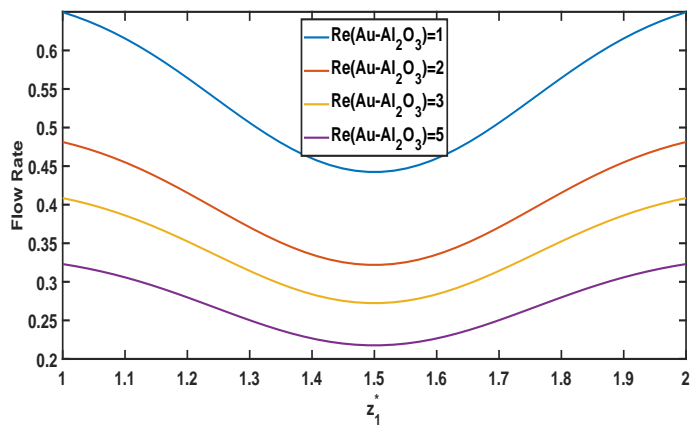

(e)

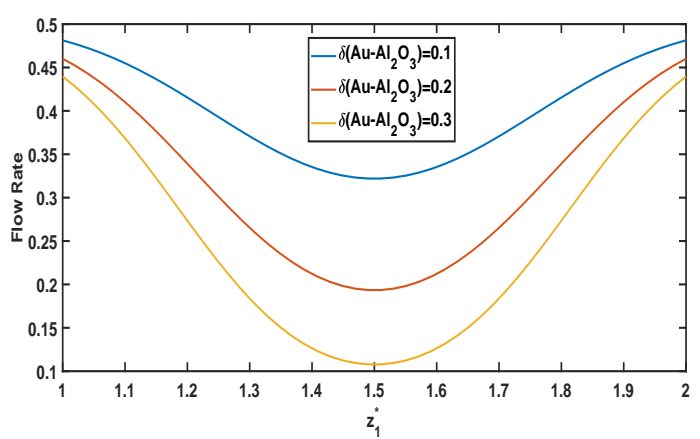

(g)

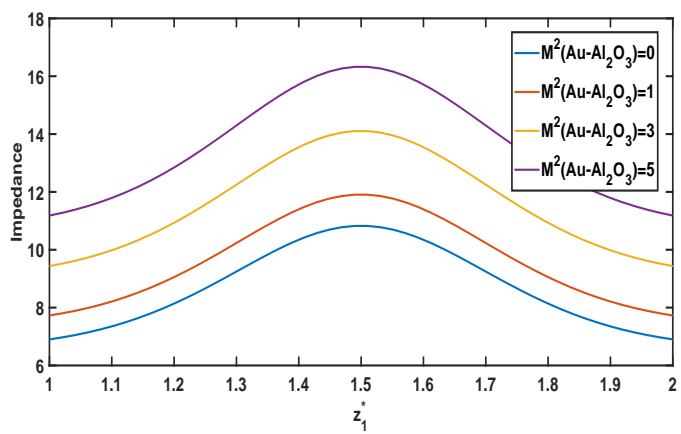

(b)

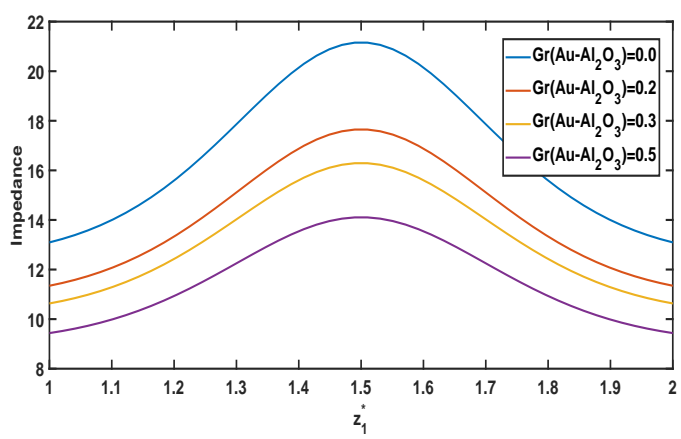

(d)

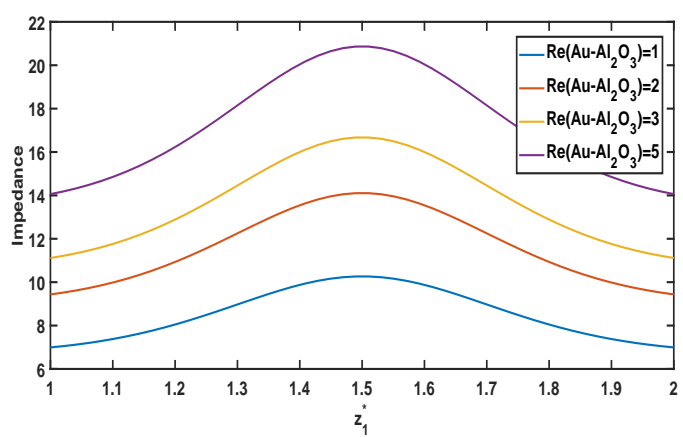

(f)

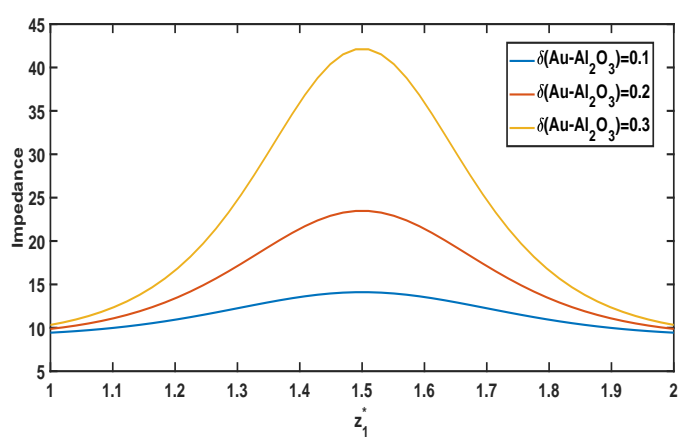

(h)

Figure 7: Effect of (a) Magnetic Number $\left(M^{2}\right)$, (b) Grashof Number $(G r)$, (c) Reynolds Number $(R e),(\mathrm{d})$ stenotic depth $(\delta)$ on Flow rate and Impedance at $t_{1}^{*}=1.3$ 
ure (7). Figure (7a) and (7b) demonstrates the influence of $M^{2}$ on flow rate and impedance profiles respectively. It is realized that when $M^{2}$ increases, the flow rate reduces due to the generation of Lorentz force, which resists the flow, and the resistive impedance increases as $M^{2}$ increases since the generation of Lorentz force increases the flow resistance. The flow rate and impedance profiles for distinct $G r$ values are illustrated in Figure (7c) and (7d). There is an increment in flow rate and declination in resistive impedance profiles with increasing $G r$ values since concentration variations of nanoparticles inside blood control the diffusion of nanoparticle species, increasing the thermal buoyancy force. This process also results in a reduction in blood flow resistance and, as a result, an increase in volumetric flow rate. Flow rate and impedance profiles for different values of $R e$ are portrayed in Figure (7e) and (7f). An increase in the values of $R e$ from 1 to 5 results in a constant suppression of flow rate, corroborating the axial velocity results computed before. Because inertial effects are more significant at higher Reynolds numbers, flow retardation and decreased flux are the primary consequences. The resistance impedance profiles rise as expected because viscous forces overcome the inertial forces. Figure $(7 \mathrm{~g})$ and $(7 \mathrm{~h})$ demonstrate the variation in flow rate with $\delta$. The flow rate drops, and the resistive impedance profiles show an increasing trend with increasing $\delta$ values because an increase in $\delta$ causes a hindrance in the blood flow near the stenotic throat, lowering the flow rate and enhancing the resistance impedance profiles.

\subsection{Heat Transfer Coefficient}

In thermodynamics and mechanics, the heat transfer coefficient, also known as the film coefficient or film effectiveness, is the proportionality constant between the heat flux and the thermodynamic driving force for the movement of heat. The effects of parameters like Eckert number $(E c)$, Prandtl number $(P r)$, and Reynolds number $(R e)$ are shown in Figure (8). Figure (8a) depicts the combined effect of $E c$ and $P r$. With a significant increase in the $E c$ and $P r$, the heat transfer coefficient displays a downward trend. Because there is a base fluid in nanoparticles, the particles travel in a crisscross pattern, causing particle collisions inside the fluid, generating additional heat. However, colliding particles produce more and more heat due to viscous dissipation, slowing the rate of heat 


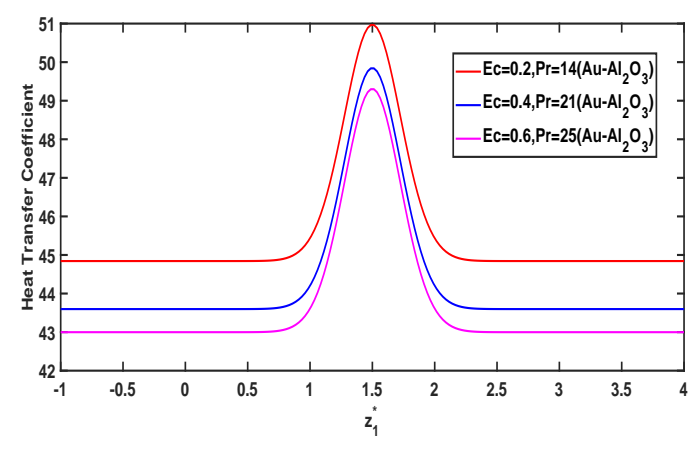

(a)

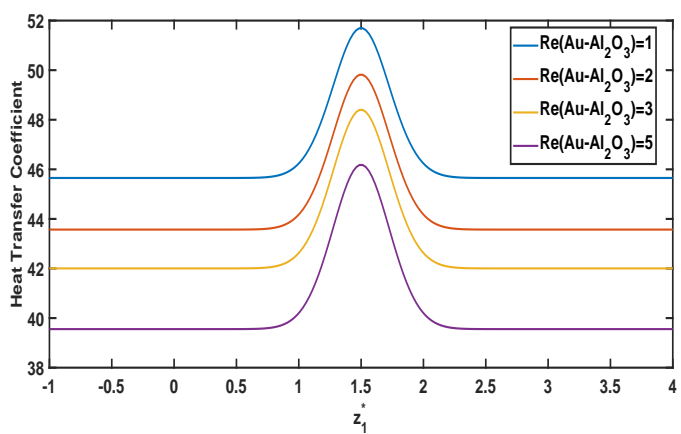

(b)

Figure 8: Influence of (a) Eckert Number $(E c)$ and Prandtl Number $(P r)$, (b) Reynolds Number $(R e)$ on Heat Transfer Coefficient at $t_{1}^{*}=1.3$

transfer and raising the temperature. In contrast to $R e$, Figure (8b) shows the profiles for heat transfer coefficient. With the enhancement in $R e$ values, a declination in heat transfer coefficient can be seen, which clearly illustrates that there is a decrease in temperature with increasing $R e$ values since low Reynolds number indicate that the viscous forces are more significant than inertial forces and agreeing with the results shown in Figure (5d).

\subsection{Velocity Contours}

The velocity contours are intriguing, and they accurately portray the research efforts. With the help of these contours, we can see what the true profiles are. These contours are depicted in Figure (9) for different values of Magnetic Number $\left(M^{2}\right)$, Darcy Number ( $\left.D a\right)$, stenotic depth $(\delta)$, different concentrations of both the nanoparticles $\left(\phi_{1}, \phi_{2}\right)$. Figures (9a-9c) show a comparison of blood flow for various values of $M^{2}$. These contours show how the strength of the magnetic field affects blood flow in both the radial and axial directions. The blood velocity is maximum when $M^{2}=1$, and it slowly decreases as $M^{2}$ value rises. It is self-evident that when the value of $M^{2}$ increases, the Lorentz force causes a hindrance in the velocity zone, which is consistent with the data in Figure (4a). Figures (9d-9f) depicts the streamline contours for various values of $D a$. As the value of $D a$ increases, so does the velocity since the resistance provided by solid fibers in the porous media is reduced as the $D a$ increases, increasing the non-dimensional blood 


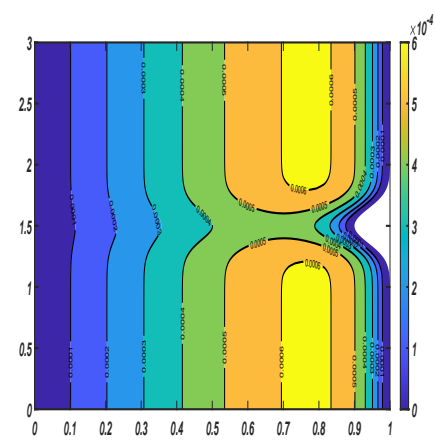

(a) $M^{2}=1$

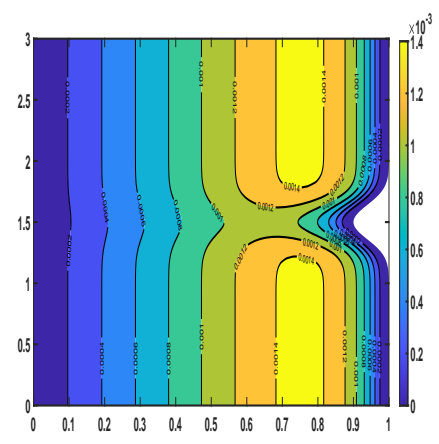

(d) $D a=0.1$

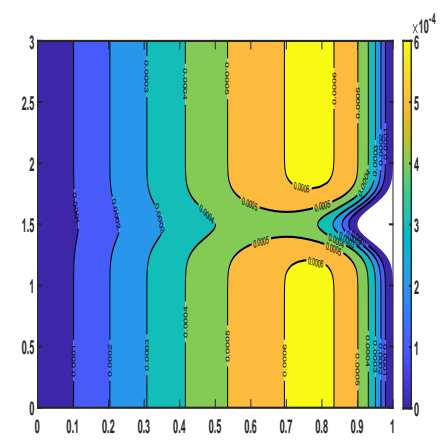

(g) $\delta=0.1$

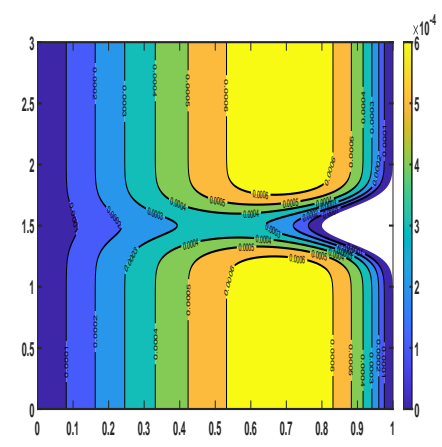

(j) $\phi_{1}=0, \phi_{2}=0$

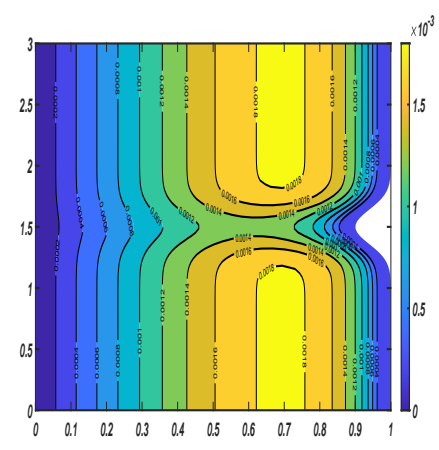

(b) $M^{2}=3$

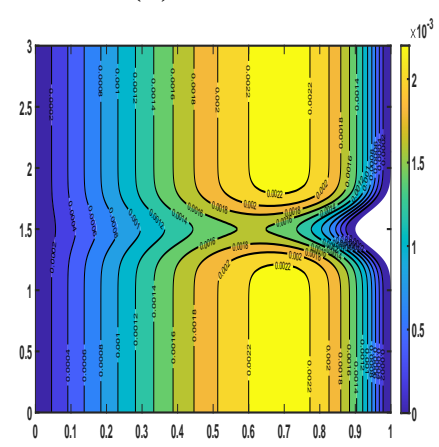

(e) $D a=1$

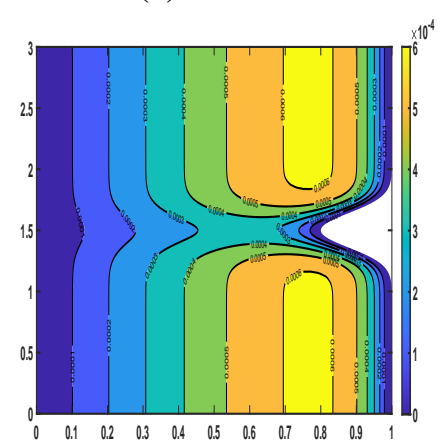

(h) $\delta=0.2$

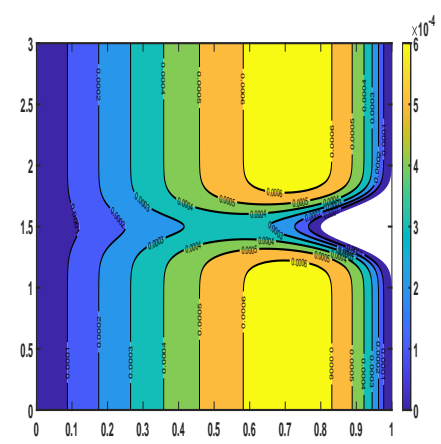

(k) $\phi_{1}=0.01, \phi_{2}=0$

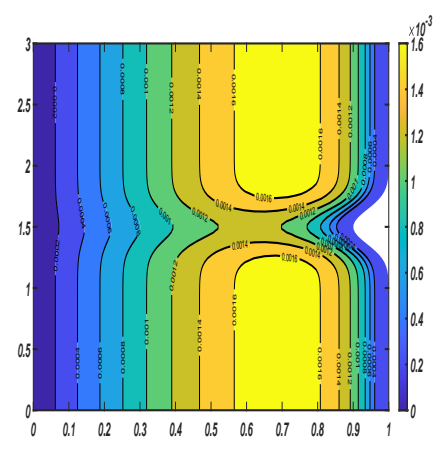

(c) $M^{2}=5$

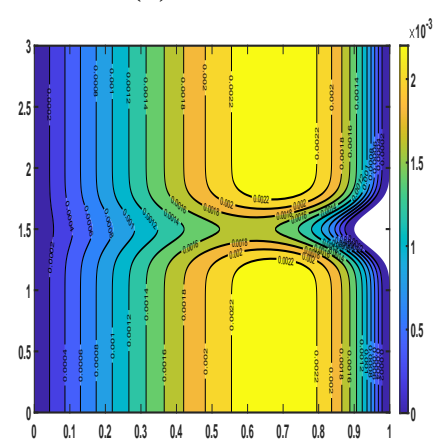

(f) $D a=2$

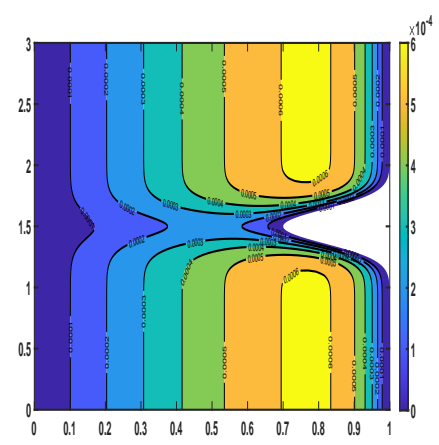

(i) $\delta=0.3$

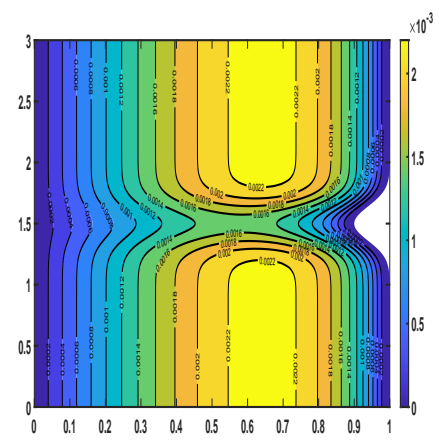

(l) $\phi_{1}=0.01, \phi_{2}=0.01$

Figure 9: Variation in blood flow patterns for various values of different parameters 
velocity as seen in Figure (4b). With increasing $D a$ values, the maximum velocity area expands as well. The contours for $\delta$ are illustrated in Figures (9g-9i). When comparing Figures (9g-9i), it can be seen that velocity is impacted at the stenotic throat $(\mathrm{z}=1.5)$ and decreases as $\delta$ increases because an increase in $\delta$ causes a hindrance in the blood flow near the stenotic throat. The effect of $\phi_{1}$ and $\phi_{2}$ on blood flow is depicted in Figure (9j-91). The pure blood instance is depicted in Figure (9j), in which no nanoparticle is added to the blood. Figure (9k) depicts the insertion of Au-nanoparticles, demonstrating that inserting the Au-nanoparticle increases blood velocity. Moreover, Figure (91) depicts the addition of $\mathrm{Al}_{2} \mathrm{O}_{3}$-nanoparticle, which demonstrates that adding the second nanoparticle reduces blood velocity.

\section{Conclusions}

This study investigates the consequences of hybrid nanoparticles on base fluid (blood) passing through an artery with mild stenosis. The hybrid nano-fluid model aims to stimulate nanoparticle medication delivery in a stenosed artery of the human circulatory system. The Crank-Nicolson approach has been used to achieve the computational solutions of unsteady coupled partial differential equations. The axial blood velocity, temperature profile, volumetric flow rate, arterial wall shear stress (WSS), resistance impedance, and heat transfer coefficient have been estimated for a wide variety of physical parameters. The key findings of the study may be summarized as follows:

- In comparison to unitary nanoparticles (Au-blood), adding hybrid nanoparticles ( $\left.\mathrm{Au}-\mathrm{Al}_{2} \mathrm{O}_{3} / \mathrm{blood}\right)$ in the blood reduces axial velocity more effectively.

- The flow rate decreases as the stenosis height increases, whereas the impedance increases because an increase in $\delta$ causes a hindrance in the blood flow near the stenotic throat, lowering the flow rate and enhancing the resistance impedance profiles.

- Streamlines depict that velocity decreases on increasing Magnetic number because with an increase in the value of $M^{2}$, the Lorentz force causes a hindrance in the velocity zone. In 
contrast, it increases with an increase in Darcy number since the resistance provided by solid fibers in the porous media is reduced.

- With an enhancement in pressure gradient parameter $\left(B_{1}\right)$, the increment in non-dimensional axial velocity is observed since the blood flow is accelerated as the pressure parameter is increased.

- The non-dimensional temperature profiles declines with an increment in both Prandtl number and Reynolds number since the heat transmission from the artery diminishes when the Prandtl number increases and viscous forces are more significant than inertial forces in case of low Reynolds number resulting in a decrement of the non-dimensional temperature profile.

- As the concentration of both nanoparticles falls, there is a declination in non-dimensional temperature profiles. In contrast, the velocity increases with an increase in the concentration of Au-nanoparticles and decreases with an increase in the concentration of $\mathrm{Al}_{2} \mathrm{O}_{3}$ nanoparticles.

- The heat transfer coefficient declines with simultaneous increment in Eckert and Prandtl number because the particles travel in a crisscross pattern, causing particle collisions inside the fluid, generating additional heat. However, colliding particles produce more and more heat due to viscous dissipation, slowing the heat transfer rate.

The present work deals with blood flow through a bell-shaped stenosed artery using $\mathrm{Au}-\mathrm{Al}_{2} \mathrm{O}_{3}$ hybrid nanoparticles suspended in the blood flow as a porous media considering temperaturedependent viscosity model. Although temperature-dependent viscosity is addressed in the current simulations, more complex non-Newtonian features of the blood are not explicitly examined. As a result, future research will look into other rheological models for magnetized blood flow. Furthermore, the shape factor effects of the nanoparticles were not considered in this investigation. Moreover, this research is limited to a bell-shaped non-tapered artery, but it could be expanded in the future to include both converging and diverging arteries. 


\section{References}

[1] Ijaz S, Nadeem S. Examination of nanoparticles as a drug carrier on blood flow through catheterized composite stenosed artery with permeable walls. Computer methods and programs in biomedicine. 2016;133:83-94.

[2] Nadeem S, Ijaz S. Theoretical examination of nanoparticles as a drug carrier with slip effects on the wall of stenosed arteries. International journal of heat and mass transfer. 2016;93:1137-1149.

[3] Eid MR, Alsaedi A, Muhammad T, Hayat T. Comprehensive analysis of heat transfer of goldblood nanofluid (Sisko-model) with thermal radiation. Results in physics. 2017;7:4388-4393.

[4] Zaman A, Khan AA, Ali N. Modeling of unsteady non-Newtonian blood flow through a stenosed artery: with nanoparticles. Journal of the Brazilian Society of Mechanical Sciences and Engineering. 2018;40(6):1-12.

[5] Ayub M, Shahzadi I, Nadeem S. A ballon model analysis with Cu-blood medicated nanoparticles as drug agent through overlapped curved stenotic artery having compliant walls. Microsystem Technologies. 2019;25(8):2949-2962.

[6] Chatterjee A, Changdar S, De S. Study of nanoparticle as a drug carrier through stenosed arteries using Bernstein polynomials. International Journal for Computational Methods in Engineering Science and Mechanics. 2020;21(5):243-251.

[7] Tripathi D, Prakash J, Tiwari AK, Ellahi R. Thermal, microrotation, electromagnetic field and nanoparticle shape effects on $\mathrm{Cu}-\mathrm{CuO} /$ blood flow in microvascular vessels. Microvascular Research. 2020;132:104065.

[8] Abo-Elkhair R, Bhatti M, Mekheimer KS. Magnetic force effects on peristaltic transport of hybrid bio-nanofluid ( $\mathrm{Au}-\mathrm{Cu}$ nanoparticles) with moderate Reynolds number: An expanding horizon. International Communications in Heat and Mass Transfer. 2021;123:105228. 
[9] Khan SU, Al-Khaled K, Bhatti M. Bioconvection analysis for flow of Oldroyd-B nanofluid configured by a convectively heated surface with partial slip effects. Surfaces and Interfaces. 2021;23:100982.

[10] Jamshed W, Nisar KS, Ibrahim RW, Shahzad F, Eid MR. Thermal expansion optimization in solar aircraft using tangent hyperbolic hybrid nanofluid: A solar thermal application. Journal of Materials Research and Technology. 2021;14:985-1006.

[11] Redouane F, Jamshed W, Suriya Uma Devi S, Prakash M, Nisar KS, Nasir NAAM, et al. Galerkin finite element study for mixed convection $\left(\mathrm{TiO}_{2}-\mathrm{SiO}_{2} /\right.$ water) hybrid-nanofluidic flow in a triangular aperture heated beneath. Scientific reports. 2021;11(1):1-15.

[12] Amine BM, Redouane F, Mourad L, Jamshed W, Eid MR, Al-Kouz W. Magnetohydrodynamics natural convection of a triangular cavity involving $\mathrm{Ag}-\mathrm{MgO} /$ water hybrid nanofluid and provided with rotating circular barrier and a quarter circular porous medium at its rightangled corner. Arabian Journal for Science and Engineering. 2021;46(12):12573-12597.

[13] Jamshed W, Prakash M, Hussain SM, Eid MR, Nisar KS, Muhammad T, et al. Entropy Amplified Solitary Phase Relative Probe on Engine Oil Based Hybrid Nanofluid. Chinese Journal of Physics. 2021;Doi: 10.1016/j.cjph.2021.11.009.

[14] Hiba B, Redouane F, Jamshed W, Saleel CA, Devi SSU, Prakash M, et al. A novel case study of thermal and streamline analysis in a grooved enclosure filled with ( $\mathrm{Ag}-\mathrm{MgO} /$ Water) hybrid nanofluid: Galerkin FEM. Case Studies in Thermal Engineering. 2021;28:101372.

[15] Alimohamadi H, Imani M, Forouzandeh B. Computational analysis of transient nonNewtonian blood flow in magnetic targeting drug delivery in stenosed carotid bifurcation artery. International Journal of Fluid Mechanics Research. 2015;42(2).

[16] Zaman A, Ali N, Sajjad M. Effects of nanoparticles $\left(\mathrm{Cu}, \mathrm{TiO}_{2}, \mathrm{Al}_{2} \mathrm{O}_{3}\right)$ on unsteady blood flow through a curved overlapping stenosed channel. Mathematics and Computers in Simulation. 2019;156:279-293. 
[17] Varmazyar M, Habibi M, Amini M, Pordanjani AH, Afrand M, Vahedi SM. Numerical simulation of magnetic nanoparticle-based drug delivery in presence of atherosclerotic plaques and under the effects of magnetic field. Powder Technology. 2020;366:164-174.

[18] Somasundaram R, Subramanyam Reddy A. Pulsating flow of electrically conducting couple stress nanofluid in a channel with ohmic dissipation and thermal radiationDynamics of blood. Proceedings of the Institution of Mechanical Engineers, Part E: Journal of Process Mechanical Engineering. 2021;p. 09544089211025177. Doi: $10.1177 /$ 09544089211025177.

[19] Snyder GK. Influence of temperature and hematocrit on blood viscosity. American Journal of Physiology-Legacy Content. 1971;220(6):1667-1672.

[20] Eckmann DM, Bowers S, Stecker M, Cheung AT. Hematocrit, volume expander, temperature, and shear rate effects on blood viscosity. Anesthesia \& Analgesia. 2000;91(3):539-545.

[21] Akbar NS, Tripathi D, Bég OA. Variable-viscosity thermal hemodynamic slip flow conveying nanoparticles through a permeable-walled composite stenosed artery. The European Physical Journal Plus. 2017;132(7):1-11.

[22] Tiwari A, Chauhan SS. Effect of varying viscosity on two-layer model of pulsatile flow through blood vessels with porous region near walls. Transport in Porous Media. 2019;129(3):721-741.

[23] Bhatti M, Abbas MA. Simultaneous effects of slip and MHD on peristaltic blood flow of Jeffrey fluid model through a porous medium. Alexandria Engineering Journal. 2016;55(2):1017-1023.

[24] Mirza I, Abdulhameed M, Shafie S. Magnetohydrodynamic approach of non-Newtonian blood flow with magnetic particles in stenosed artery. Applied Mathematics and Mechanics. 2017;38(3):379-392. 
[25] Abdollahzadeh Jamalabadi MY, Daqiqshirazi M, Nasiri H, Safaei MR, Nguyen TK. Modeling and analysis of biomagnetic blood Carreau fluid flow through a stenosis artery with magnetic heat transfer: A transient study. PLoS One. 2018;13(2):e0192138.

[26] Khentout A, Kezzar M, Sari MR, Ismail T, Tich Tich MS, Boutelba S, et al. The electrical magnetohydrodynamic (MHD) and shape factor impacts in a mixture fluid suspended by hybrid nanoparticles between non-parallel plates. Proceedings of the Institution of Mechanical Engineers, Part E: Journal of Process Mechanical Engineering. 2021;p. 09544089211057971. Doi: $10.1177 / 09544089211057971$.

[27] Jha B, Gwandu B. MHD free convection flow in a vertical porous super-hydrophobic microchannel. Proceedings of the Institution of Mechanical Engineers, Part E: Journal of Process Mechanical Engineering. 2021;235(2):470-478.

[28] Tripathi B, Sharma BK, Sharma M. Modeling and analysis of mhd two-phase blood flow through a stenosed artery having temperature-dependent viscosity. The European Physical Journal Plus. 2019;134(9):466.

[29] Tripathi B, Sharma BK. Influence of Heat and Mass Transfer on Two-Phase Blood Flow with Joule Heating and Variable Viscosity in the Presence of Variable Magnetic Field. International Journal of Computational Methods. 2020;17(03):1850139.

[30] Tripathi B, Sharma BK. Two-phase analysis of blood flow through a stenosed artery with the effects of chemical reaction and radiation. Ricerche di Matematica. 2021;p. 1-27.

[31] Bég OA, Bhargava R, Rawat S, Halim K, Takhar HS. Computational modeling of biomagnetic micropolar blood flow and heat transfer in a two-dimensional non-Darcian porous medium. Meccanica. 2008;43(4):391-410.

[32] Sharma B, Mishra A, Gupta S. Heat and mass transfer in magneto-biofluid flow through a non-Darcian porous medium with Joule effect. Journal of Engineering Physics and Thermophysics. 2013;86(4):766-774. 
[33] El-dabe NT, Moatimid GM, Hassan MA, Mostapha DR. Effect of partial slip on peristaltic flow of a Sisko fluid with mild stenosis through a porous medium. Appl Math. 2016;10(2):673-687.

[34] Sheikholeslami M. Numerical approach for MHD Al2O3-water nanofluid transportation inside a permeable medium using innovative computer method. Computer Methods in Applied Mechanics and Engineering. 2019;344:306-318.

[35] Eid MR, Al-Hossainy A, Zoromba MS. FEM for blood-based SWCNTs flow through a circular cylinder in a porous medium with electromagnetic radiation. Communications in Theoretical Physics. 2019;71(12):1425.

[36] Bhatti M, Arain M, Zeeshan A, Ellahi R, Doranehgard M. Swimming of Gyrotactic Microorganism in MHD Williamson nanofluid flow between rotating circular plates embedded in porous medium: Application of thermal energy storage. Journal of Energy Storage. 2021;p. 103511. Doi: $10.1016 / j$.est.2021.103511.

[37] Zhang L, Bhatti M, Shahid A, Ellahi R, Bég OA, Sait SM. Nonlinear nanofluid fluid flow under the consequences of Lorentz forces and Arrhenius kinetics through a permeable surface: A robust spectral approach. Journal of the Taiwan Institute of Chemical Engineers. 2021;124:98-105.

[38] Bilal M, Ramzan M, Mehmood Y, Kbiri Alaoui M, Chinram R. An entropy optimization study of non-Darcian magnetohydrodynamic Williamson nanofluid with nonlinear thermal radiation over a stratified sheet. Proceedings of the Institution of Mechanical Engineers, Part E: Journal of Process Mechanical Engineering. 2021;235(6):1883-1894.

[39] Bose S, Datta A, Ganguly R, Banerjee M. Lagrangian magnetic particle tracking through stenosed artery under pulsatile flow condition. Journal of Nanotechnology in Engineering and Medicine. 2013;4(3):031006. 
[40] Chakravarty K, Dalal D. A two-phase model for drug release from microparticles with combined effects of solubilisation and recrystallisation. Mathematical biosciences. 2016;272:2433.

[41] Ponalagusamy R, Priyadharshini S. A numerical model on pulsatile flow of magnetic nanoparticles as drug carrier suspended in Herschel-Bulkley fluid through an arterial stenosis under external magnetic field and body force. International Journal of Computer Mathematics. 2019;96(9):1763-1786.

[42] Dinarvand S, Nademi Rostami M, Dinarvand R, Pop I. Improvement of drug delivery microcirculatory system with a novel pattern of $\mathrm{CuO}-\mathrm{Cu} /$ blood hybrid nanofluid flow towards a porous stretching sheet. International Journal of Numerical Methods for Heat \& Fluid Flow. 2019;29(11):4408-4429.

[43] Misra J, Shit G. Blood flow through arteries in a pathological state: A theoretical study. International Journal of Engineering Science. 2006;44(10):662-671.

[44] Ahmed A, Nadeem S. Effects of magnetohydrodynamics and hybrid nanoparticles on a micropolar fluid with 6-types of stenosis. Results in physics. 2017;7:4130-4139.

[45] Shit G, Majee S. Pulsatile flow of blood and heat transfer with variable viscosity under magnetic and vibration environment. Journal of Magnetism and Magnetic Materials. 2015;388:106-115.

[46] Tripathi J, Vasu B, Bég OA. Computational simulations of hybrid mediated nanohemodynamics (Ag-Au/Blood) through an irregular symmetric stenosis. Computers in Biology and Medicine. 2021;130:104213.

[47] Das S, Pal T, Jana R. Outlining Impact of Hybrid Composition of Nanoparticles Suspended in Blood Flowing in an Inclined Stenosed Artery Under Magnetic Field Orientation. BioNanoScience. 2021;11(1):99-115. 
[48] Ellahi R, Raza M, Vafai K. Series solutions of non-Newtonian nanofluids with Reynolds' model and Vogel's model by means of the homotopy analysis method. Mathematical and Computer Modelling. 2012;55(7-8):1876-1891.

[49] Venkatesan G, Reddy AS. Insight into the dynamics of blood conveying alumina nanoparticles subject to Lorentz force, viscous dissipation, thermal radiation, Joule heating, and heat source. The European Physical Journal Special Topics. 2021;p. 1-11.

[50] Burton AC. Physiology and Biophysics of the Circulation. Academic Medicine. 1965;40(8):xxx-Xxxvi.

[51] Priyadharshini S, Ponalagusamy R. Mathematical modelling for pulsatile flow of Casson fluid along with magnetic nanoparticles in a stenosed artery under external magnetic field and body acceleration. Neural Computing and Applications. 2019;31(3):813-826.

[52] Smith GD, Smith GD, Smith GDS. Numerical solution of partial differential equations: finite difference methods. Oxford university press; 1985.

[53] Anderson JD, Wendt J. Computational fluid dynamics. vol. 206. Springer; 1995.

[54] Zaman A, Ali N, Kousar N. Nanoparticles $\left(\mathrm{Cu}, \mathrm{TiO}_{2}, \mathrm{Al}_{2} \mathrm{O}_{3}\right)$ analysis on unsteady blood flow through an artery with a combination of stenosis and aneurysm. Computers \& Mathematics with Applications. 2018;76(9):2179-2191.

[55] Katritsis D, Kaiktsis L, Chaniotis A, Pantos J, Efstathopoulos EP, Marmarelis V. Wall shear stress: theoretical considerations and methods of measurement. Progress in cardiovascular diseases. 2007;49(5):307-329. 